\title{
POETICA VERBALE E FIGURATIVA DELLE DUE CITTÀ NOTE SUL FIRENZE, BML, PLUT. 12.17*
}

\author{
Elisa BRILLI \\ Fondation de la Maison de Sciences de l'Homme \\ \& Laboratoire d'études sur les Monothéismes, EPHE, Paris
}

\section{L'efficacia retorica e poetica delle due città agostiniane}

Poche rappresentazioni culturali hanno conosciuto una fortuna pari a quella della civitas come immagine della comunità e poche dottrine possono vantare più seguaci della dottrina agostiniana delle due città. Le ragioni di tale successo sono senza dubbio molteplici e spaziano dall'auctoritas del Padre della Chiesa alla versatilità di queste rappresentazioni. Tra queste ragioni, tuttavia, occorre tenere in conto anche la dimensione retorica e poetica. Il fatto di ritrarre la comunità degli eletti sotto forma di una civitas perfetta, la civitas Dei in cui è trasposta la Gerusalemme biblica, così come il fatto di visualizzare la storia del creato come il confronto tra questa civitas, pellegrina nel mondo in conseguenza del peccato originale e destinata a trionfare nell'altro, e la sua antagonista, la civitas terrena (o diaboli, definizione già agostiniana e prediletta nel medioevo), avvinta alla mondanità e avviata a una sicura rovina nell'aldilà, è «commovente». Lo è etimologicamente, nello stesso senso cioè in cui la retorica antica suggeriva che dovessero essere le immagini prescelte dal retore per conquistare l'attenzione, il favore e la memoria del pubblico.

${ }^{*}$ Lo studio presentato in quest' articolo ha beneficiato dei preziosi consigli di Chiara Frugoni, Jérôme Baschet, Didier Méhu, Jean-Claude Schmitt e Brian Stock, oltre che dei suggerimenti e commenti dei partecipanti al seminario del "Groupe de recherche sur les pouvoirs et les sociétés de l'Occident médiéval et moderne» (presso l'Université du Québec à Montréal), coordinato da Michel Hébert e Piroska Nagy, e dei membri del seminario di dottorato del «Laboratoire d'études sur les Monothéismes» (presso l'EPHE di Parigi), coordinato da Olivier Boulnois, in occasione di due presentazioni orali (rispettivamente, nel settembre del 2012 e nell'aprile del 2013). A loro vanno i miei più sinceri ringraziamenti e le scuse per le lacune ancora presenti. Questo lavoro è stato reso possibile dalla borsa post-doc Fernand Braudel-IFER/Incoming della Fondation de la Maison de Sciences de l'Homme di Parigi (co-finanziata dall'Unione Europea) accordata al progetto Imagining the City of God. Visual Interpretations of St Augustine's De civitate Dei (12 $12^{\text {th }} 15^{\text {th }}$ century). 
È lo stesso Agostino, il cui debito nei confronti della sua prima formazione retorica è troppo noto per attardarvisi ${ }^{1}$, a sottolineare l'importanza di questa dimensione. Agostino la presenta anzi come oggettiva, inerente cioè alla creazione stessa e non solo alla dottrina che egli andava elaborando per descriverla. L'antitesi -osservava a proposito della caduta degli angeli ribelli- è una figura retorica che assicura l'armonia del discorso; allo stesso modo, si deve credere che, nei piani del Creatore, la creazione includa il male ai fini di una più sottile armonia, alla stregua di un bellissimo poema ${ }^{2}$. In questa eloquentia, che Agostino vuole rerum e non solo verborum, la contrapposizione delle due città, figura di antitesi per eccellenza, è deputata a rappresentare quella tra Bene e Male; e in essa Agostino convoglia molteplici dualità che strutturano l'orizzonte teologico cristiano.

Innanzitutto, la tensione tra tempo ed eternità, ossia tra le incertezze e le sofferenze del divenire dell'hoc saeculum ('questo mondo/tempo') e la perfezione che il fedele crede essere propria del mondo inaugurato dalla seconda parousia e dall'ultimo giudizio. La civitas terrena (o diaboli) appartiene ottusamente alla storicità, poiché non riconosce altra realtà oltre la

\footnotetext{
${ }^{1}$ Oltre al classico Henri-Irénée Marrou, Saint Augustin et la fin de la culture antique (1938), suivi de la Retractatio (1949), Paris, E. de Boccard, $1958^{4}$, cfr. il volume collettivo, incentrato sulle Confessioni, Augustine, from Rhetor to Theologian, ed. Joanne McWilliam, Timothy Barnes, Michael Fahey and Peter Slater, Toronto, Wilfrid Laurier University Press, 1992 e i saggi raccolti nella terza parte di The Rhetoric of St. Augustine of Hippo, De doctrina christiana and the search for a distinctly Christian rhetoric, ed. Richard Leo Enos, Robert Thompson et al., Waco (Tx), Baylor University Press, 2008.

${ }^{2}$ Cfr. Aug. Hipp., De civ. Dei XI 18, ed. B. Dombart e A. Kalb, in Corp. Christ. Ser. Lat. 47-48, Turnhout 1955, vol. 48, p. 337, 11. 1-18: «Neque enim Deus ullum, non dico angelorum, sed uel hominum crearet, quem malum futurum esse praescisset, nisi pariter nosset quibus eos bonorum usibus commodaret, atque ita ordinem saeculorum tamquam pulcherrimum carmen ex quibusdam quasi antithetis honestaret. Antitheta enim quae appellantur, in ornamentis elocutionis sunt decentissima, quae latine appellantur opposita, uel quod expressius dicitur, contraposita. [...] Sicut ergo ista contraria contrariis opposita sermonis pulchritudinem reddunt: ita quadam non uerborum, sed rerum eloquentia contrariorum oppositione saeculi pulchritudo componitur» (trad. it. di Domenico Gentili, Roma, Città Nuova, 2002² , p. 549: «Inoltre Dio non creerebbe non dico un angelo ma neanche un uomo, di cui avesse previsto che sarebbe divenuto malvagio, se non conoscesse pure a quale profitto dei buoni destinarli e ornare così mediante antitesi, come se fosse un bellissimo poema, la vicenda dei tempi. Quelle che si chiamano antitesi sono molto opportune nell'eleganza del discorso. In latino si dicono opposizioni o, per tradurre più esplicitamente, contrapposizioni.[...] Come dunque questi contrari opposti ai propri contrari rendono l'armonia del discorso, così l'armonia della vicenda dei tempi è data dall'opposizione dei contrari in un determinato discorso non di parole ma di fatti»). La stessa metafora della creazione come poema nel quale i contrari si armonizzano, si ritrova in Aug. Hipp., Ep. 138, 5 e 166, 13. Secondo Agostino, la Scrittura riflette questo piano divino: vi si rende dunque conto delle malefatte della città negativa per meglio far risaltare il progresso della divina (cfr. De civ. Dei XV 8, 1, ed. cit., vol. 48, p. 463, 1. 10: «ita ut nec de altera societate hominum taceretur [...] ut ciuitas Dei etiam suae aduersariae comparatione clarescat»; trad. it. cit., p. 755: «Però non si doveva passar sotto silenzio l'altra umana società, che consideriamo la città terrena, nell'esclusivo intento di farne menzione affinché la città di Dio risplenda anche nel confronto con la sua avversaria»). Per alcuni esempi della fortuna dell'antitesi nella letteratura cristiana dei primi secoli e una riflessione sul suo uso aperta alla ricerca iconologica, cfr. Antonio Quacquarelli, «L'antitesi retorica», in Vetera Christianorum, 19:2 (1982), pp. 223-237.
} 
propria; la civitas Dei, invece, i cui membri sono pure immersi nel divenire in conseguenza del peccato originale, transita in questo mondo senza però appartenervi e il suo iter di pellegrina è fiduciosamente orientato al trascendente. Questa tensione di natura ontologica tra la frammentarietà e precarietà dell'essere nel tempo e la sua pienezza fuori dal tempo, si combina allora con una tensione di carattere etico-escatologico, quella tra chi è avvinghiato alla mondanità e chi invece sa guardare oltre di essa. In questa prospettiva, Agostino definisce la civitas terrena (o diaboli) come la comunità metastorica di coloro che si sono votati all'amor sui e che, dimentichi di Dio, saranno da lui dimenticati nell'aldilà; la civitas Dei corrisponde invece alla comunità metastorica di coloro che hanno correttamente coltivato l'amor Dei, ossia dei futuri eletti. Per questa via le due città agostiniane assimilano e risemantizzano la ricca serie dei binomi paolini, si pensi ai celebri carnale/spirituale, generazione/rigenerazione, natura/grazia e, non da ultimo, terreno/celeste.

Accanto a queste dualità principali, le due città agostiniane ne sintetizzano anche altre. Quella, ad esempio, tra stato politico (che è il significato primo del sintagma 'civitas terrena' nel latino dell'età di Agostino) e comunità religiosa. Si tratta di una tensione di lunga data che rimonta alle persecuzioni contro i primi cristiani, ma essa risulta non meno importante dopo la conversione dell' $I m-$ pero romano al cristianesimo. In questo contesto, e tanto più dopo il sacco di Roma (410 d.C.) e la conseguente recrudescenza della polemica anti-cristiana da parte del partito pagano, emerge l'esigenza di ri-articolare il rapporto tra stato politico e comunità religiosa in modo tale da, al contempo, escludere la loro sovrapposizione e garantire un margine di collaborazione. Attraverso l'immagine delle due città, Agostino sviluppa ora una polemica senza quartiere contro l'ideologia statuale pagana; ora consiglia, insistendo invece sul tema della permixtio ('mescolanza') delle due città in questo mondo, pazienza e sopportazione ai fedeli sottoposti al potere politico. Radicalità dei principi e flessibilità delle loro applicazioni si combinano mirabilmente nell'azione politica del vescovo di Ippona e, a riprova della finezza delle categorie da lui coniate, nella tradizione medievale le due città continueranno ad assimilare le tensioni che travagliano la Cristianità, mutando di volta in volta la loro fisionomia al contatto con le nuove configurazioni storico-politiche alle quali furono applicate.

Senza bisogno di dettagliare oltre queste note introduttive ${ }^{3}$, è chiaro che l'efficacia delle due città è anche di natura retorica e poetica. Essa risiede nella capacità sintetica di quest'immagine discorsiva tramite la quale Agostino

${ }^{3}$ Per maggiori precisioni mi permetto di rinviare a Elisa Brilli, Una vicina città. Storia del paradigma della «civitas diaboli» nell'Occidente medievale, tesi di dottorato in cotutela, La Sapienza-Roma (dir. G. Inglese) e École des Hautes Études en Sciences Sociales-Paris (dir. J.-C. Schmitt), 2009. 
convoglia dualità e tensioni di tipo e di matrici diversi. Conseguentemente, l'antitesi delle due città possiede una grande versatilità e si presta, nel discorso agostiniano così come nel medievale, a essere impugnata in forme e in vista di obiettivi assai diversi: ora sottolineando come l'una -la civitas terrena (o diaboli)- sarà oltrepassata dall'altra -la civitas Dei-, quando interessa mettere in luce la transitorietà della condizione umana e sulla base di quella confortare l'aspirazione cristiana al suo superamento; ora enfatizzando la loro radicale opposizione etico-escatologica, quando si tratta di stigmatizzare l'attitudine corrotta degli amanti del saeculum e di rivendicare la giustizia divina; ora, insistendo sulla permixtio delle due città in questo mondo, quando s'intende predicare alla comunità dei fedeli la pazienza, la sopportazione e, nella misura del possibile, la cooperazione in attesa della fine dei tempi.

La capacità sintetica e la versatilità dell'immagine discorsiva delle due città non sono tuttavia tali da non sollevare alcune difficoltà di ordine tanto logico-concettuale quanto teologico. Uno dei modi per coglierle è analizzare le trasposizioni di quest'immagine in media differenti dal discorso verbale e, più precisamente, di considerare le sue mises en image. Le soluzioni iconografiche e gli accorgimenti messi in opera dagli artisti medievali per trasporre l'antitesi delle due città dal discorso verbale al figurativo rivelano infatti quali significazioni, fra le varie che questa figura discorsiva possiede, sono più a cuore nei diversi contesti di elaborazione delle immagini materiali, ma anche i suoi limiti ossia la sua equivocità, dal momento che le significazioni delle due città si spingono ben oltre la semplice polisemia.

\section{Un esempio di mise en image: Firenze, BML, Plut. 12.17}

La complessità della mise en image del De civitate Dei e dei concetti in esso sviluppati è ben testimoniata da un esemplare miniato del trattato, oggi conservato alla Biblioteca Medicea-Laurenziana di Firenze sotto la segnatura Plutei $12.17^{4}$. Realizzato tra gli anni Venti e gli anni Trenta del XII secolo,

\footnotetext{
${ }^{4}$ Membr.; $348 \times 250 \mathrm{~mm}$ (specchio della scrittura: 340 x 245); fols. I + 226; scritto su 2 colonne, su 45 linee, in minuscola carolina attribuita a più mani. Si vedano André Wilmart, «La tradition des grands oeuvrages de S. Augustin, III. La Cité de Dieu», in Miscellanea Agostiniana, vol. II. Studi Agostiniani, Roma, Tipografia poliglotta Vaticana, 1931, pp. 279-294, in particolare p. 284, n. ${ }^{\circ} 89$; M. Oberleitner, Italien, Wien, Österreichischen Akademie der Wissenschaften, 1969-70 (coll. «Die handschriften Überlieferung der Werke des heiligen Augustinus», I/1-2), 1, p. 32, n. ${ }^{\circ} 13$; Biblioteca Medicea Laurenzia$n a$, ed. Antonietta Morandini et alii, Firenze, Centro internazionale del Libro-Nardini Editore, 1986, pp. 96-97, tav. XLIX-LI; Umanesimo e Padri della Chiesa. Manoscritti e incunaboli di testi patristici da Francesco Petrarca al primo Cinquecento, (Firenze, BML, 5 febbraio 9 agosto 1997), Milano, Rose ed., 1997, n. ${ }^{\circ}$ 82, pp. 319-325; Gli Umanisti e Agostino. Codici in mostra, ed. Daniela Coppini e Mariangela Regogliosi, Firenze, Polistampa, 2001, scheda n. ${ }^{\circ}$ 46, pp. 193-194, tavv. E-F e 48-49. Per 
questo manoscritto è generalmente attribuito, su base paleografica e stilistica, a Canterbury e forse allo scriptorium dell'abbazia benedettina di Saint Augustine (di Canterbury) ${ }^{5}$-sebbene alcuni studiosi abbiano sottolineato la presenza di elementi stilistici caratteristici piuttosto dello scriptorium della cattedrale, Christ Church-ed è stata proposta la sua identificazione con uno degli esemplari che figurano in una lista antica dei codici posseduti della biblioteca di Saint Augustine ${ }^{6}$.

Questo manoscritto ha più volte attirato l'attenzione dei ricercatori per diversi motivi. Si tratta ad esempio di uno dei tredici testimoni delle Notae di Lanfranco di Pavia al trattato di Agostino ${ }^{7}$, e di uno dei più antichi esemplari miniati di quest'opera ${ }^{8}$. Sin dagli studi di Meyer Schapiro, il Plut. 12.17 è inoltre spesso menzionato dagli storici dell'arte quale esempio della transizione stilistica dalla maniera anglo-sassone a quella successiva alla conquista normanna ${ }^{9}$. Non da ultimo, essendo pervenuto alla biblioteca medicea forse già negli anni di Cosimo il Vecchio (1389-1464) e certamente al tempo di Pietro de' Medici (1416-1469), del quale è la nota di possesso che figura al fol. 226r («Liber Petri de Medecis Cos. Fil.»), questo codice si è prestato alla

altra bibliografia cfr. passim.

${ }^{5}$ Più cauta la valutazione attributiva in sede paleografica, cfr. Richard Gameson, «English Manuscript Art in the Late Eleventh Century: Canterbury and its Context», in Canterbury and the Norman Conquest: Churches, Saints and Scholars, 1066-1109, ed. Richard Eales and Richard Sharpe, London, Hambeldon Press, 1995, pp. 95-144, in part. p. 128 e nota 123, e Id., The Manuscripts of Early Norman England (1066-1130), London, Oxford University Press, 1999, p. 89, n. 299.

${ }^{6}$ Cfr. St Augustine's Abbey, Canterbury, ed. B. C. Barker-Benfield, London, British Library, 2008 , «Corpus of British Medieval Library Catalogues», 13/1-3, vol. 1, p. 525 e vol. 3, p. 2090.

${ }^{7} \mathrm{Al}$ f. 226r, ma interrotte (inc.: «Sententia quam beatus Augustinus...»; expl. «... in na vero desinentia denominativa», cfr. Margaret Gibson, «Lanfranc's Notes on Patristic Texts», in Journal of Theological Studies, n.s., 33 (1971), pp. 435-448, a p. 437 (sul Plut. 12.17) e pp. 439-440 (edizione del testo). Su questo stesso foglio figura inoltre l'enigmatica Coniectura Bedae.

${ }^{8} \mathrm{Su}$ questa tradizione cfr. Alexandre de Laborde, Les manuscrits à peinture de la Cité de Dieu, 3 vols., Paris, E. Rahir, 1909 e Kenneth B. Steinhauser, «Manuscripts», in Augustine through the Ages: an Encyclopedia, ed. Allan D. Fitzgerald, Cambridge, Wm. B. Eerdmans, 1999 , pp. 525-533, da integrare, per il periodo più antico, con Susanne Wittekind, «Die Illustration von Augustinustexten im Mittelalter», in Der Kommentar in Antike und Mittelalter, ed. Wilhelm Geerlings - Christian Schulze, 2 voll., Leiden, Brill, 2002-2004, vol. II, pp. 101-127 e Alessandro Cosma, Valerio Da Gai e Gianni Pittiglio, Iconografia agostiniana, I. Dalle Origini al XIV secolo, Roma, Città Nuova, 2011 («Opera omnia di sant'Agostino», 41). Per l'analisi di altri esemplari del XII secolo, mi permetto di rinviare a Elisa Brilli, «Lire le $D e$ civitate Dei par le biais de l'Apocalypse et vice-versa. Contribution à l'étude du ms. Oxford, Bodleian Library, Laud Misc. 469», in Quand l'image relit le texte. Regards croisés sur les manuscrits médiévaux, éd. par Sandrine Hériché-Pradeau et Maud Perez-Simon, Paris, Presses de la Sorbonne Nouvelle, 2013, pp. 139-158, e a Elisa Brilli, «As Formas da História. A doutrina agostiniana das seis idades do mundo e algumas de suas visualizações no século XII», in Revista de História, 165: 2 (2011), dossiê: A Imagem medieval: história e teoria, pp. 121-150.

${ }^{9}$ Meyer Schapiro, «From Mozarabic to Romanesque in Silos», in Art Bulletin, 21:4 (1939), p. 314 e nota 4. Per un' analisi in questa chiave, cfr. Charles Reginald Dodwell, The Canterbury School of Illumination 1066-1200, Cambridge, Cambridge University Press, 1954, pp. 28, 35, 64, 122. 
revisione del testo condotta dai primi umanisti, come indicano le tracce di diverse mani quattrocentesche, tra le quali quella dell' intellettuale fiorentino Niccolò Niccoli (1364-1464) $)^{10}$.

Nonostante la celebrità, le quattro tavole miniate che aprono questo manoscritto, benché spesso citate singolarmente, hanno raramente costituito l'oggetto di un'indagine globale, volta a comprendere il loro discorso alla luce della lettura del De civitate Dei che doveva essere propria alla comunità dalla quale e per la quale questa sontuosa copia era stata approntata ${ }^{11}$. È ciò che quest'articolo si propone di fare nella prospettiva più generale di una riflessione sulla mise en image dei concetti agostiniani, dopo aver analizzato i soggetti di ogni tavola singolarmente e discusso le interpretazioni che ne sono state offerte finora.

\subsection{Dalla storia al giudizio individuale post mortem (fol. 1v) [fig. 1]}

I tre registri della prima miniatura, apposta al verso del primo foglio, sono inquadrati da una cornice che si presenta, nella parte inferiore, decorata da formelle a motivi vegetali e, nella parte superiore, sormontata da un'arcata a tutto sesto. La giuntura tra una parte e l'altra è assicurata da due elementi ornamentali collocati a due terzi del registro mediano -un dettaglio, come si vedrà, degno di nota in questo ciclo che accorda una speciale attenzione al dispositivo della cornice e alle sue infrazioni.

Il registro inferiore ritrae una scena di lavoro rurale. Dei due personaggi incappucciati, il primo tiene il timone di un aratro a versoio, mentre, con l'altra mano, tenta forse di liberare il coltro dal terreno ${ }^{12}$; il secondo, sprona uno

\footnotetext{
${ }^{10}$ Per un'analisi di quest'aspetto cfr. Cosimo il Vecchio de' Medici 1389-1464. Essays in Commemoration of the $600^{\text {th }}$ Anniversari of Cosimo de' Medici's Birth, with an introduction by E. Gombrich, ed. Francis Ames-Lewis, Oxford, Clarendon, 1992, in part. p. 126, nota 47 e p. 149, nota 52, per l'identificazione della mano di Niccolò Niccoli che si deve ad Albinia C. de La Mare); Gli Umanisti e Agostino. Codici in mostra, ed. Daniela Coppini e Mariangela Regogliosi, Firenze, Polistampa, 2001, scheda n. ${ }^{\circ}$ 46, pp. 193-194, tavv. E-F e 48-49 e Xavier E. J. D. B. van Binnebeke, «Per la biblioteca di Cosimo e Lorenzo de' Medici e la produzione di manoscritti a Firenze nel primo Rinascimento», in Rinascimento, 2 s. 41 (2001), pp. 199-223.

${ }^{11}$ Con la sola eccezione di Kenneth B. Steinhauser, «Augustin moralisé. Some observations on Florence, Laurenziana, Plut. 12, cod. 17», in Augustine in iconography: history and legend, ed. by Joseph C. Schnaubelt and Frederick Van Fleteren, New York, Peter Lang, 1999, pp. 577-593, sul quale cfr. infra.

${ }^{12} \mathrm{Cfr}$. Perrine Mane, Le travail à la campagne au Moyen Âge. Etude iconographique, Ouvrage publié avec le concours du Centre national du livre, Paris, A. \& J. Picard, 2006, p. 129 (e sull'iconografia medievale dell'aratura: pp. 104-120). L'assenza del pezzo di raccordo tra le due parti dell'aratro s'imputerà a una distrazione del miniatore e potrebbe essere indizio del fatto che copiasse da un modello, come suggerito da Angela Dillon Bussi, «Come i miniatori onorarono Sant'Agostino (Miniature nei codici agostiniani di biblioteche fiorentine)», in Gli Umanisti e Agostino. Codici in mostra, ed. Donatella Coppini e Mariangela Regogliosi, Firenze, Polistampa, 2001, pp. 55-72, a p. 69.
} 
dei due buoi aggiogati, mentre sullo sfondo e così in primo piano sul terreno è schizzato con tratto leggero un gran numero di uccelli.

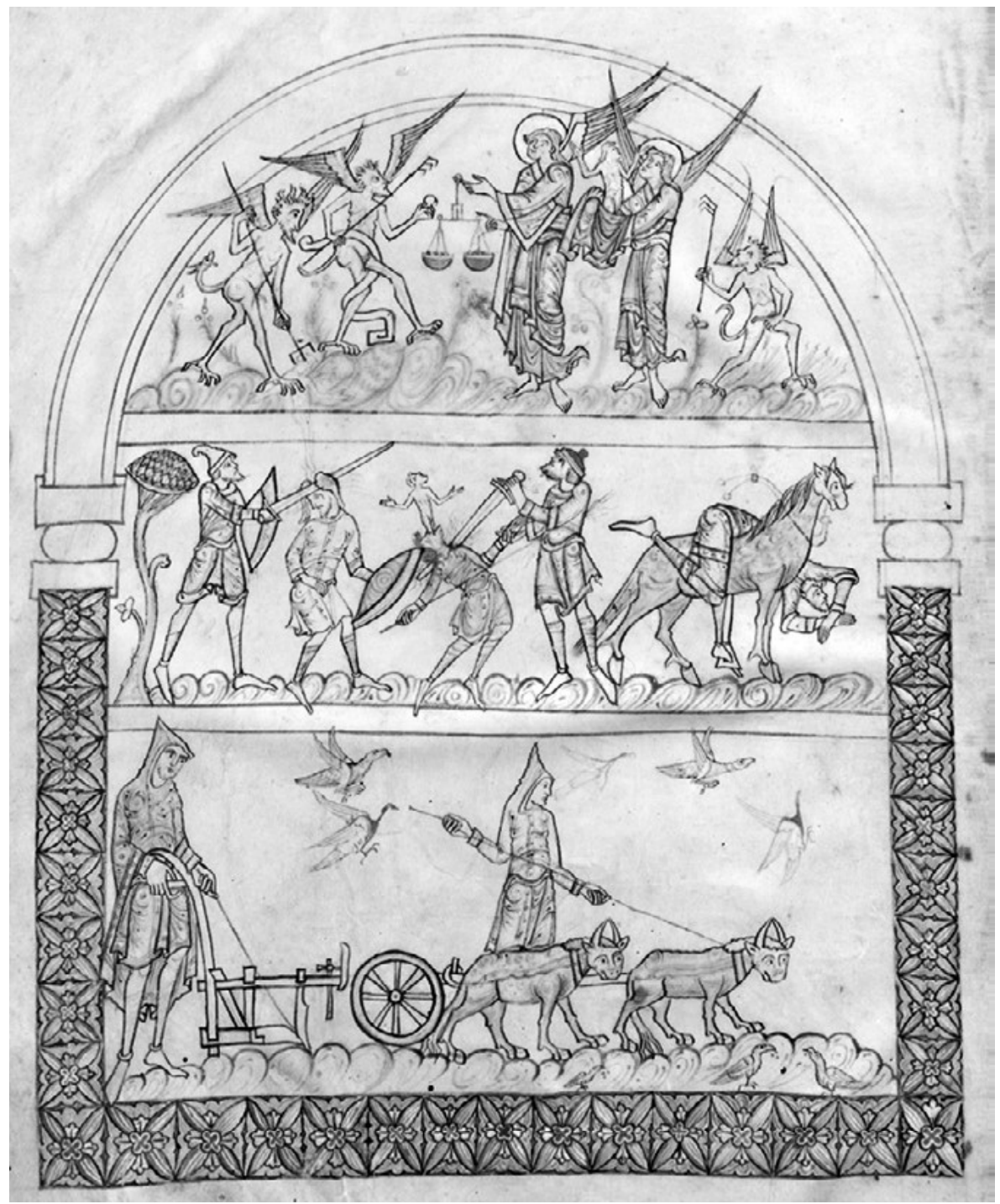

Fig. 1: Firenze, Biblioteca Medicea-Laurenziana, ms Plut 12.17, fol. 1v. (C) Biblioteca Medicea-Laurenziana.

Nel secondo registro si susseguono tre scene d'intenso dinamismo.

Da sinistra a destra, una prima coppia di milites, entrambi muniti di scudo, è ritratta nell'istante in cui uno dei due squarta con la spada il cranio dell'altro, l'anima del quale, improvvisamente liberata, s'innalza sino al registro 
superiore (nelle riproduzioni scarsamente visibile per la cattiva resa fotografica dell'inchiostro rosso). A seguire, altri due armati si trucidano a vicenda: il primo, piegato all'indietro ad arco e trapassato alla giugulare dalla spada dell'avversario, esala l'anima e insieme infilza il nemico con la lancia, come manifesta il fiotto di sangue che zampilla dalla schiena di costui. Infine, un terzo personaggio, un altro cavaliere a giudicare dal ricco ornamento della veste, cade rovinosamente da cavallo. L'insieme dei dettagli di quest'ultima scena -le mani incrociate per proteggere il viso, un piede ancora impigliato nella staffa, le zampe anteriori del cavallo stese e impuntate al suolo, le briglie fluttuanti a mezz'aria- insiste sulla puntualità dell'istante che precede l'impatto. L'ultimo registro accoglie invece una psicostasia, o giudizio individuale delle anime post mortem. Da un lato e dall'altro di una bilancia a due piatti, l'angelo pesatore (l'arcangelo Michele?) stende l'indice destro verso un diavolo che, con un obolo (o un sasso) in una mano e il forcone nell'altra, gli indirizza uno sguardo impotente, forse per aver scoperto di aver perduto l'anima che già è accolta esultante nelle braccia di un secondo angelo. Agli estremi della scena, altri due diavoli: il primo, dalla coda ibridata, arpiona l'anima del (con licenza) «cavaliere dimezzato» del registro inferiore, mentre l'altro solleva vanamente il forcone contro l'anima eletta. Il diavolo con l'obolo è inoltre avvinghiato da un oggetto poco chiaro, forse un cartiglio male interpretato. L'ambientazione campestre della psicostasia, in assenza di una vulgata medievale sul luogo in cui dovrebbe darsi il giudizio individuale post mortem, merita di essere segnalata.

Quest'immagine assembla dunque alcuni soggetti singolarmente ben diffusi -l'aratura; la caduta da cavallo, memore probabilmente dell'iconografia della superbia secondo la Psychomachia di Prudenzio; la psicostasia- in una composizione priva di paralleli e di significato non immediatamente trasparente. La maggioranza degli studiosi ha voluto vedere nei primi due registri la rappresentazione di due soggetti allegorici contrapposti, identificati per lo più con l'opposizione pace/guerra e con quella buono/cattivo governo ${ }^{13}$,

\footnotetext{
${ }^{13}$ Quest'interpretazione, proposta da Hanns Swarzenski, Monuments of Romanesque art, the art of church treasures in North-Western Europe, 2nd ed, London 1967, tav. 87, è seguita da Claus Michael Kauffmann, Romanesque Manuscripts 1066-1190, London, Harvey Miller, 1975, pp. 62-63, no. 19, tavv. $49,50,56$ e ha proliferato nella letteratura secondaria. Si vedano a titolo di esempio gli usi da parte di Madeline Harrison Caviness, «Conflicts Between Regnum and Sacerdotium as reflected in a Canterbury Psalter of ca. 1215», in Art Bulletin, 61: 1 (1979), pp. 38-58, a p. 48; Chiara Frugoni, Una lontana città. Sentimenti e immagini nel Medioevo, Torino, Einaudi, 1983, p. 194 e ill. 99; Cynthia Hahn, «Peregrinatio et Natio: The Illustrated Life of Edmund, King and Martyr», in Gesta, 30: 2 (1991), pp. 119-139, a p. 129; Claire Richter Sherman, Imaging Aristotle. Verbal and Visual Representation in Fourteenth-Century France, University of California Press, Berkeley-Los Angeles-London 1995, p. 196; Elisabeth Den Hartog, «All Nature Speaks to God All Nature Teaches Man. The Iconography of the Twelfth-century 
eventualmente combinati con l'opposizione laboratore/bellatores ${ }^{14}$, nonché talvolta ricondotti all'immagine agostiniana delle due civitates.

In verità, quest' ipotesi di lettura è tutt'altro che piana. Se l'associazione di lavoro agricolo e pace (nel primo registro) è possibile, perché nel luogo deputato a ritrarre la guerra (nel registro mediano) è stato inserito un personaggio vittima di un incidente banale, anzi topico, piuttosto che di un fatto d'armi? A sua volta, il rinvio all'opzione buono/cattivo governo non nasconde la proiezione retrospettiva del soggetto ritratto da Ambrogio Lorenzetti nella sala dei Nove del Palazzo pubblico di Siena, ma né gli elementi iconografici né quelli contestuali supportano l'estensione di questo dualismo nell'ambito della cultura monastica dell'Inghilterra meridionale dell'inizio del XII secolo e, del resto, la stessa esecuzione lorenzettiana è supportata, in un contesto ben più avvezzo a tali categorie, da un grande sfoggio di rubriche e cartigli a chiarimento delle allegorie effigiate. Rispetto al tentativo di riferirsi per questa via alle due città agostiniane (secondo una parabola associativa del tipo: aratura $\rightarrow$ pace $\rightarrow$ buon governo $\rightarrow$ civitas Dei e, viceversa, cavalieri $\rightarrow$ guerra $\rightarrow$ cattivo governo $\rightarrow$ civitas terrena o diaboli), vale la pena ricordare che le due città non corrispondono tout court, né in Agostino né nella tradizione medievale, a due modelli politici di governo terreno. Si aggiungerà poi che la collocazione dei soggetti che gli studiosi suppongo significare la civitas Dei nel registro più basso appare inusuale a fronte della sintassi simbolica dello spazio miniato medievale, che riserva in genere la posizione infima ai soggetti di minore valore. Credo invece prezioso il riferimento ai tipi socio-politici di laboratores e bellatores, e indubbiamente i personaggi rappresentati nei due registri presentano tali connotazioni, a condizione tuttavia di non assolutizzare questa coppia poiché lo schema medievale è abitualmente tripartito e gli oratores (o chierici) che lo completano sono omessi nella prima miniature e invece dislocati nelle seguenti.

Mi sembra dunque preferibile la spiegazione proposta da Angela Dillon Bussi che ha suggerito di vedere in questa miniatura una rappresentazione della terrestrità e dell'immanenza fino al momento dell'exitus da questa dimensione, qui fatta corrispondere al trapasso, e alla comparsa in giudizio dell'anima individuale ${ }^{15}$. A differenza delle precedenti, quest'interpretazione

Capitals in the Westwork Gallery of the Church of St. Servatius in Maastricht», in Zeitschrift für Kunstgeschichte, 59 (1996), pp. 29-62, alle pp. 35-36 e fig. 6; Steinhauser, "Augustin moralisé...», art. cit., p. 581.

${ }^{14}$ Così Michael Camille, «Labouring for the Lord: The Ploughman and the Social Order in the Luttrell Psalter», in Art History, 10: 4 (1987), pp. 423-454, a p. 431 e fig. 8.

${ }^{15}$ Dillon Bussi, «Come i miniatori onorarono Sant'Agostino...», art. cit., pp. 68-9: «il loro assieme [dei registri] mira a rappresentare la vicenda dell'uomo, puntualizzandone i tre momenti essenziali, cioè 
non suggerisce alcuna opposizione tra i primi due registri -ciò che, del resto, mal si accorda con la struttura tripartita dell'immagine- e legge invece la loro serie in modo continuo dall'alto in basso. In favore di questa lettura si possono inoltre avanzare alcuni elementi ulteriori. Si ricorderà innanzitutto che i calendari medievali aprono abitualmente il ciclo annuale con il soggetto iconografico dell'aratura, caratteristico del mese di gennaio, come testimoniano bene alcuni esempi anglosassoni provenienti forse entrambi da Canterbury [fig. 2, fig. 3]. Il soggetto del primo registro potrebbe allora essere stato suggerito da questo riferimento, dall'associazione cioè corrente dell'aratura con il ciclo dei lavori annuali e dunque con lo scorrere del tempo ordinario e ciclico.

È notevole poi l'insistenza del secondo registro sull'istante della morte e più precisamente sul momento del trapasso dell'anima colta, come si è visto, nel suo distaccarsi dal corpo. A questo proposito, si può ricordare che Agostino discute lungamente di tali problemi nel XIII libro del trattato, soffermandosi specialmente, nei capitoli 9 e 11, sull'adynaton linguistico costituito dall'espressione «morente». Non si può essere al tempo stesso in morte e in vita e da ciò discende -argomenta Agostino- l'impossibilità di cogliere precisamente il momento del trapasso che, come il presente nella speculazione sviluppata nelle Confessiones, non può che sfuggire all'osservazione ${ }^{16}$. Il secondo registro sembra appunto voler rendere visibile il complesso e paradossale rapporto che si dà tra morte e vita, tra dimensione corporea e spirituale, e l'importanza di tale articolazione è amplificata dal dispositivo della cornice che a quest'altezza segna uno stacco.

vita terrena (cui corrisponde la cornice simbolica della Natura), morte terrena, vita eterna ultraterrena (cui corrisponde la cornice simbolica del Cielo), e ponendo l'accento sul momento del trapasso da un mondo all'altro, quello cioè che rivela la sua duplice natura, materiale e spirituale (un passaggio che figurativamente si traduce nel mutare della cornice)».

${ }^{16}$ Cfr. Aug. Hipp., De civ. Dei XIII 11, 1, ed. cit., vol. 48, p. 393, 11. 11-20: «Quando itaque sit moriens, id est in morte, ubi neque sit vivens, quod est ante mortem, neque mortuus, quod est post mortem, sed moriens, id est in morte, difficillime definitur. Quamdiu quippe est anima in corpore, maxime si etiam sensus adsit, procul dubio vivit homo, qui constat ex anima et corpore, ac per hoc adhuc ante mortem, non in morte esse dicendus est; cum vero anima abscesserit omnemque abstulerit corporis sensum, iam post mortem mortuusque perhibetur. Perit igitur inter utrumque, quo moriens vel in morte sit; quoniam si adhuc vivit, ante mortem est; si vivere destitit, iam post mortem est. Numquam ergo moriens, id est in morte, esse comprehenditur» (trad. italiana cit., p. 648: «È assai difficile circoscrivere il limite di tempo in cui un tale sia morente, cioè nella morte, e perciò non sia vivente perché lo è prima della morte, né morto perché lo è dopo la morte, ma morente, cioè nella morte. Finché l'anima è nel corpo, soprattutto se permane la facoltà sensitiva, indubbiamente l'uomo, il quale è composto di anima e di corpo, ancora vive. Si deve perciò considerare prima della morte, non nella morte. Quando invece l'anima si sarà allontanata e avrà sottratto totalmente la facoltà sensitiva, l'uomo è dopo la morte ed è considerato morto. Scompare fra l'uno e l'altro lo stato per cui è morente, cioè nella morte perché, se ancora vive è prima della morte, se ha cessato di vivere è dopo la morte. Dunque non si può mai ritenere che è morente, cioè nella morte»). Il riferimento è a Confessiones XI, 15, 18-20. 


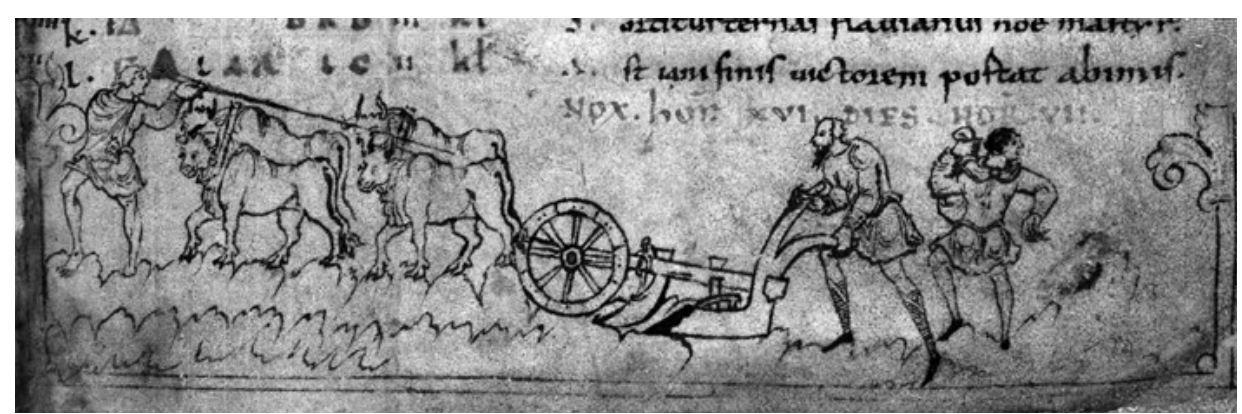

Fig. 2: British Library, Cotton Julius ms A.VI, fol. 3r (Canterbury, Christ Church?, XI sec. in.). C) British Library.

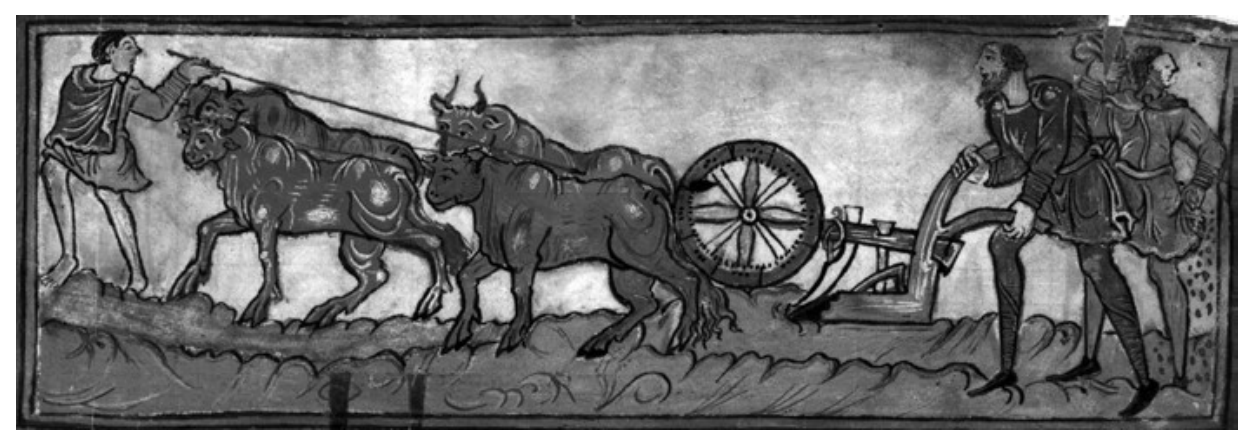

Fig. 3: British Library, Cotton Tiberius ms B.V, fol. 3r (Winchester o Canterbury, XI sec., II quarto). (C) British Library.

Diversamente, la scena effigiata nel registro superiore è indipendente dalla speculazione agostiniana circa il destino ultramondano delle anime. La trattazione di tali problemi nel De civitate Dei è tutta orientata a contestare la dottrina platonica della psicostasia, in modo da assicurare la dimensione anche corporea della resurrezione cristiana alla fine dei tempi. La scelta del soggetto della pesa rispecchia invece la rappresentazione dell'accesso all'aldilà che va definendosi tra XI e XII secolo; una rappresentazione nella quale, pur senza ridurre l'importanza del giudizio universale, il giudizio individuale delle anime post mortem acquista una visibilità crescente ${ }^{17}$. Vale poi precisare che se non è agostiniano il riferimento alla psicostasia al quale generazioni di storici dell'arte hanno ricondotto l'emergenza di questo soggetto iconografico nell'Occidente medievale, questa attribuzione erronea sembra essere

\footnotetext{
${ }^{17}$ Sulla questione cfr. Jérôme Baschet, «Jugement de l'âme, jugement dernier: contradiction, complémentarité, chevauchement?», in Revue Mabillon, n.s., 6, 67 (1995), pp. 159-203 (nel quale la miniatura del Plut. 12.17 è cit. a p. 176) e gli sviluppi in Id., «Une image à deux temps. Jugement Dernier et jugement des âmes au Moyen Âge», Images Re-vues [En ligne], hors-série $1 \mid 2008$, mis en ligne le 01 juin 2008, consulté le 20 novembre 2012. URL: http://imagesrevues.revues.org/878.
} 
solo moderna, poiché le attestazioni medievali non rinviano ad Agostino ma piuttosto alla patristica greca ${ }^{18}$. Nel medioevo, questo soggetto insomma non è associato ad Agostino neanche per via di apocrifi, e la sua scelta sarà da intendersi come un «aggiornamento» indipendente dal testo che ci si proponeva di illustrare.

Globalmente considerata, dunque, la prima miniatura può essere descritta come una rappresentazione della terrestrità e della temporalità (figurata tramite il soggetto convenzionale nei calendari dell'aratura) fino al suo esaurimento (la morte fisica e il trapasso dell'anima) e al giudizio individuale post mortem (la psicostasia).

\subsection{La civitas Dei trionfante (fol. 2v). [fig. 4]}

Il soggetto della seconda miniatura, apposta al verso del secondo foglio, pone meno problemi. La sua cornice architetturale rinvia alla Gerusalemme celeste della visione apocalittica attribuita a san Giovanni -in ragione delle pietre preziose che la ornano-, ma anche e al contempo a uno spazio ecclesiale -come indicano le quattro guglie (le più alte sormontate da croci) e l'articolazione interna che evoca la rappresentazione delle navate - $\mathrm{e}$ al paradiso terrestre- cui allude il cherubino che con la spada infuocata a guardia della porta d'accesso, sotto l'arcata centrale del registro inferiore, e i quattro fiumi che da questa porta scorrono perdendosi nel margine inferiore del foglio miniato ${ }^{19}$.

\footnotetext{
${ }^{18}$ Mi riferisco al brano «Erit enim ibi sine dubio compensatio bonorum malorumque, et velut in statera posita utraque pars. Quae demerserit, illa sibi eorum quo momentum vergitur, operarium vindicabit. Si ergo malorum multitudo superaverit, operarium suum pertrahit ad gehennam: si vero majora fuerint opera bonorum, summa vi obsistent, et repugnabunt malis, atque operatorem suum ad regionem vivorum ex ipso etiam gehennae confinio revocabunt» tradizionalmente attribuito ad Agostino, trascritto con alcuni errori e invocato come pezza d'appoggio negli studi sull'iconografia della psicostasia. Per la genesi dell'erronea attribuzione, cfr. la nota di Jean-Pierre Torrel, «Saint Augustin et la pesée des âmes ou les avatars d'une citation apocryphe», in Revue d'études augustiniennes et patristiques, 27:1-2 (1981), pp. 100-104. Per le attestazioni medievali, oltre a Auc. Inc., Sermo de symbolo xIx, in Patr. Lat. 40, coll. 1189-1202, in part. col. 1202 («Erit enim ibi sine dubio compensatio bonorum malorumque [...] ex ipso etiam gehennae confinio revocabunt»), già allegato da Torrel (a p. 102, note 8 e 9), questo stesso brano è incluso in Rabanus Maurus, De videndo Deum, de puritate cordis et modo poenitentiae, III, cap. 22, in Patr. Lat. 102, coll. 1328D-1329A e in alcuni testimoni della Collectio canonum hibernensis, dove si trova attribuito a «Iohannes metropolitanus» ossia Damasceno (così sia nel ms. Oxford, Bodleian Library, Hatton 42, ff. 129v-130r, proveniente dalla Bretagna e datato variamente al IX o X sec.; sia nel ms. Roma, Biblioteca Vallicelliana, tom. XVIII, f. 136v, proveniente dall'Italia meridionale e datato all'xI, secondo le indicazioni di Roger E. Reynolds, «The Influence of Eastern Patristic Fathers on the Canonical Collections of South Italy in the Eleventh and Early Twelfth Centuries», in Canon Law, Religion, and Politics: "Liber Amicorum" Robert Somerville, ed. Uta-Renate Blumenthal, CUA Press, 2012, pp. 75-106, in part. Appendix 2, p. 92 e Appendix 3, p. 93).

${ }^{19}$ Sottolinea il sincretismo tra città di Dio celeste e Paradiso terrestre Joaquin Yarza Luaces, «La geografia dell'aldilà», in Uomo e spazio nell'alto Medioevo: Settimane di studio del Centro italiano di
} 
Poetica verbale e figurativa delle due citTÀ

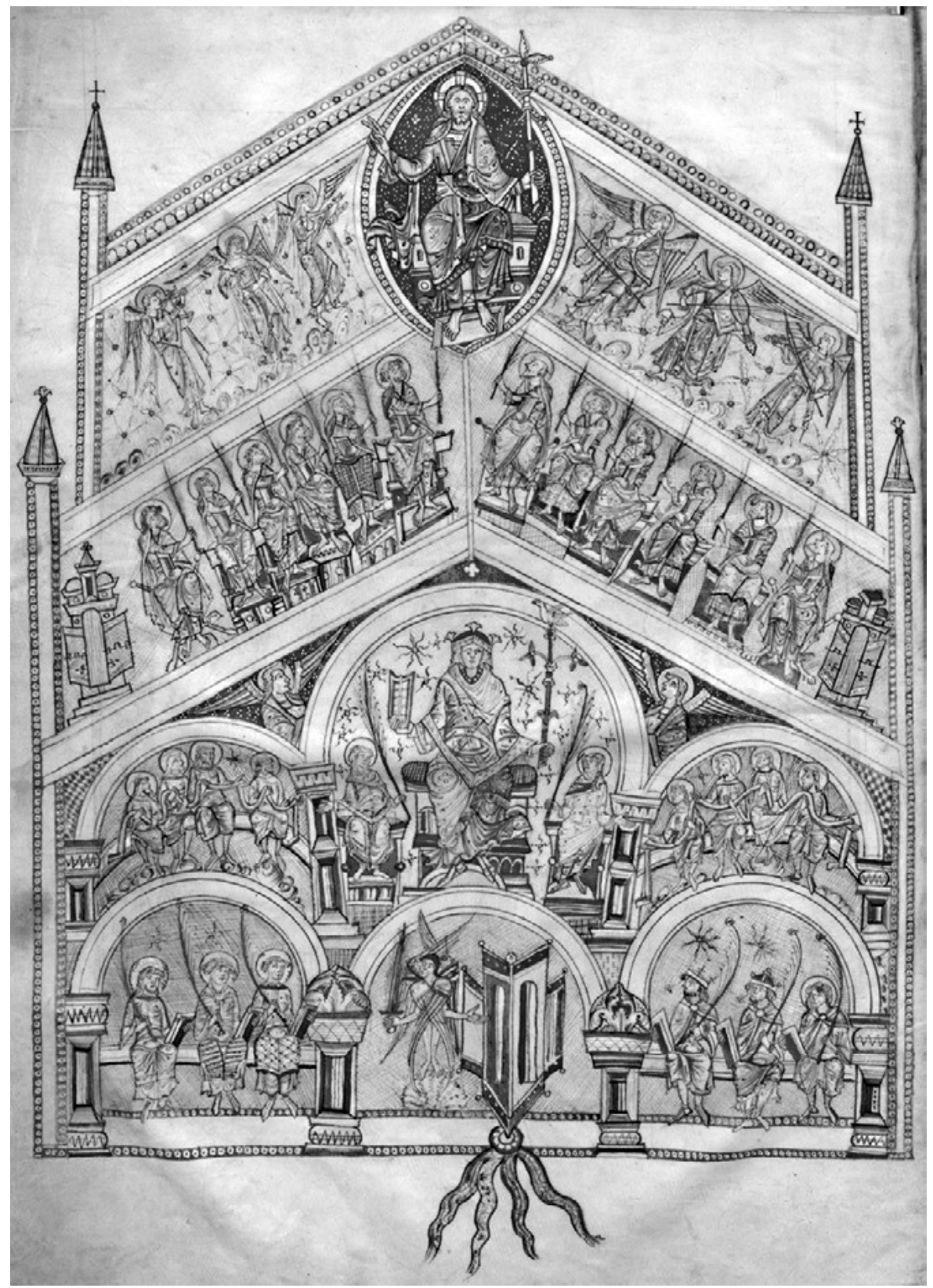

Fig. 4: Firenze, Biblioteca Medicea-Laurenziana, Plut. 12.17, fol.2v. c. Biblioteca Medicea-Laurenziana.

studi sull'alto Medioevo L (4-8 aprile 2002), Spoleto, Presso la sede del Centro, 2003, vol. I, pp. 193-235 e 17 tavv., in particolare pp. 223-224 e fig. 25. 
Immediatamente sopra alla porta, sotto l'arcata corrispondente del registro superiore, una figura femminile coronata, con manoscritto e scettro fiorito sormontato da una colomba, è correntemente intesa come un'allegoria della Ecclesia. Questa figura è ad ogni modo ritratta in modo da rinviare anche all'allegoria della Sapienza -della quale sono attributi distintivi il manoscritto e lo scettro-e alla Vergine -alla quale sembrano invece alludere sia la vegetalizzazione dello scettro sia la colomba quale figura dello Spiritus Domini che si poserà sulla discendenza di Jesse secondo la profezia di Isaia $11,1-2^{20}-$. L'asse verticale che unisce la porta d'accesso e la prosopopea femminile è quindi coronato, nel registro superiore, dalla mandorla che ospita il Cristo benedicente, secondo un tipo di maiestas Domini ben attestato nell'arte insulare di quest'epoca ${ }^{21}$. Gli altri personaggi che compongono questa scena corale risultano invece estratti liberamente dalla gerarchia della civitas Dei (dall'alto verso il basso): due gruppi di tre martiri sotto le arcate laterali, contraddistinti dalle palme e in due casi coronati ${ }^{22}$; due gruppi di quattro profeti, come sembrano indicare i volumina che sostengono, sotto le arcate corrispondenti del registro successivo, e due vergini che, velate, sono ritratte ai lati delle figura allegorica femminile dell'arcata centrale; quindi la serie dei dodici apostoli, con lo sguardo rivolto verso il Cristo; infine, la serie degli angeli musici.

Più volte citata come termine di paragone per l'iconografia di monumenti romanici $\mathrm{i}^{23}$, è chiaro che questa rappresentazione attinge a piene mani all'asse analogico e metonimico proprio alla civitas Dei-ecclesia nella cultura agostiniana e medievale, qui peraltro complicata dalla sede in cui tale immagine è eseguita, un codice del De civitate Dei. Rappresentazione trionfale della destinazione ultramondana della città di Dio o, ma è dir lo stesso, dell'ecclesia e dell'assise dei beati in quanto corpo di Cristo, quest'immagine si vuole anche effigie dell'argomento del trattato e innesca così una mise en abime caratteristica di tutta la tradizione miniata di quest'opera.

\footnotetext{
${ }^{20}$ Come suggerito da Marie-Louise Thérel, A l'origine du décor du portail occidental de NotreDame de Senlis Le triomphe de la Vierge-Église, Sources historiques, littéraire et iconographiques, Paris, Éditions du CNRS, 1984, pp. 180, 236, 333 e fig. 75.

${ }^{21}$ A titolo di esempio cfr. il Cristo del Cambridge, Trinity College, ms. B.15.34, fol. $1 r$ (Ingh., XI s.), riprodotto da Dodwell, The Canterbury School of Illumination ..., op . cit., tav. 24b (che segnala quest' immagine come possibile modello di altre simili del XII s.).

${ }^{22}$ Cfr. Elizabeth A. R. Brown e Michael W. Cothren, «The Twelfth-Century Crusading Window of the Abbey of Saint-Denis: Praeteritorum enim recordatio futurorum est exhibitio», in Journal of the Warburg and Courtauld Institutes, 49 (1986), pp. 1-40, a p. 13 per altri esempi.

${ }^{23} \mathrm{Si}$ veda ad esempio Otto Pächt, «The Illustrations of St Anselm's Prayers and Meditations», in Journal of the Warburg and Courtauld Institutes, 29 (1956), pp. 68-83, che a p. 81, nota 3 suggeriva che tale struttura potesse essere ispirata alle facciate romaniche tipiche, in particolare, della regione di Pavia, o più recentemente Pamela Tudor-Craig, Wells Cathedral West front and the City of God, Donington, Shaun Tyas, 2004, p. 370, nota 53.
} 


\subsection{Agostino e i (falsi) sapienti (fols. 3v-4r). [fig. 5, fig. 6]}

Diversamente dalle prime due, le altre due miniature che compongono la serie richiedono una lettura congiunta, tanto in ragione della disposizione affrontata quanto dei soggetti rappresentati. La prima miniatura è accolta nuovamente da una cornice architetturale connotata come ecclesiale. Nel corpo centrale, il ritratto in scranno di sant' Agostino, tonsurato e in ricchi paramenti episcopali, lo rappresenta nell'atto di mostrare un codice aperto con il corpo lievemente rivolto verso la sua sinistra e l'indice destro sollevato in un gesto forse di ammonizione e insegnamento. La taglia più piccola così come i gesti dei due gruppi di tonsurati, abbigliati secondo la moda normanno-bizantina ${ }^{24}$, sotto le arcate laterali, sottolineano la loro reverenza nei confronti del vescovo di Ippona, verso il quale i due più interni sollevano una mano. Si potrà poi suggerire che il diverso trattamento dello sfondo, uniformemente tinteggiato di verde nelle arcate laterali e invece rosso e impreziosito da disegni geometrici e da due ricche rotae nel corpo centrale, intendesse suggerire la diversa importanza dei personaggi effigiati, se non addirittura la loro appartenenza a sfere diverse: ancora del tutto mondana quella dei monaci e già celeste quella del santo nimbato.

Venendo alla miniatura antistante, la sua cornice architetturale non presenta nessun elemento ecclesiale e si distingue invece per la serie di sedici medaglioni che ospitano altrettanti ritratti che la orna [fig. 6]. Si tornerà nel seguito su questo dettaglio. Nei due registri, sono stati effigiati dei sapienti e, si direbbe, i due momenti successivi di una medesima disputa. Nel registro superiore, il gruppo, ancora parzialmente coeso in ragione del volumen tenuto dispiegato e degli sguardi tutti rivolti alla miniatura antistante, sembra già abitato da una frattura appena delineatasi: spicca infatti il vuoto simbolico dell'asse centrale - del quale si è ben visto il rilievo nelle miniature precedenti e che anche qui è evocato, al modo di un'aspettativa disattesa, dalla disposizione simmetrica degli elementi architettonici-e il braccio levato che viene così poco elegantemente a occuparlo. Nel registro inferiore la frattura sembra ormai consumata. Tre personaggi sono orientati verso la loro destra e il primo della fila persino oltrepassa con la gamba e un braccio la cornice, quasi intendesse passare nell'immagine contigua, mentre il resto del gruppo si allontana nella direzione opposta, i codici ormai abbassati.

Le trasgressioni speculari delle cornici, oltre che significativi del «love for transcending movement» tipico dell'arte anglo-sassone e ancora presente

\footnotetext{
${ }^{24} \mathrm{Cfr}$. Timothy Dawson, «Concerning an Unrecognised Tunic from Eastern Anatolia», in Byzantion, 73 (2003), pp. 201-210, a p. 209, nota 23.
} 
Elisa BRILLI

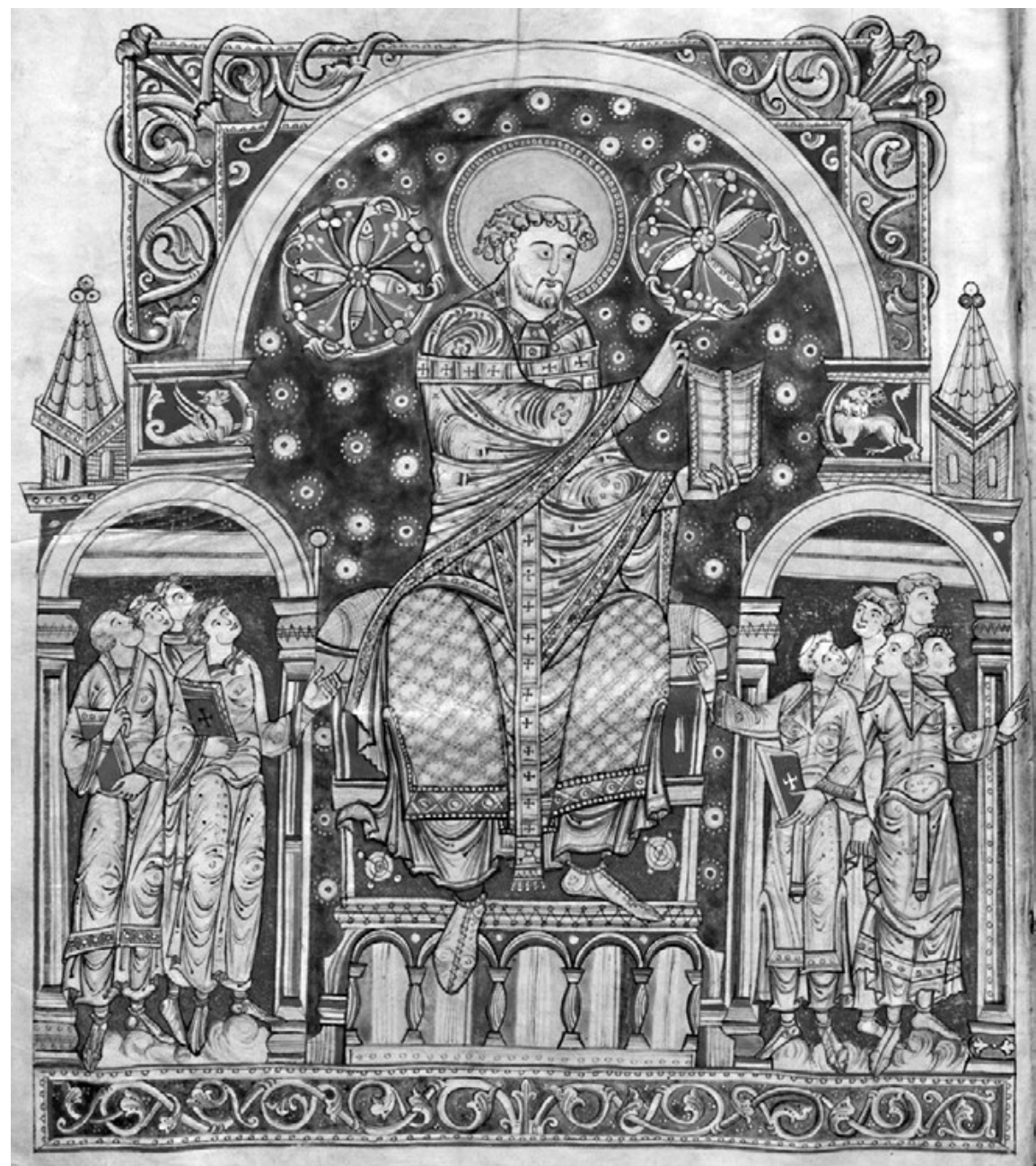

Fig. 5: Firenze, Biblioteca Medicea-Laurenziana, Plut. 12.17, fol. 3v (C) Biblioteca Medicea-Laurenziana.

in questo documento ${ }^{25}$, testimoniano innanzitutto della solidarietà dei soggetti rappresentati. Tenendo il manoscritto aperto, la doppia pagina mostra in effetti una sorta di predicazione di Agostino tanto ai monaci della prima miniatura quanto ai laici della seconda, ciò che, si può ipotizzare prestando un'intenzione narrativa ai due registri del fol. 4r, provoca una discussione tra costoro e quindi la separazione tra chi, forse convinto dal vescovo, gli si rivolge e gli altri che, volto di dosso, partono nella direzione opposta ${ }^{26}$.

\footnotetext{
${ }^{25}$ Riprendendo un'espressione di Pächt, «The Illustrations of St Anselm's Prayers and Meditations...», art. cit., p. 80, nota 1 .

${ }^{26}$ Sottolineano opportunamente il nesso tra le due miniature Steinhauser, «Augustin moralisé...»,
} 


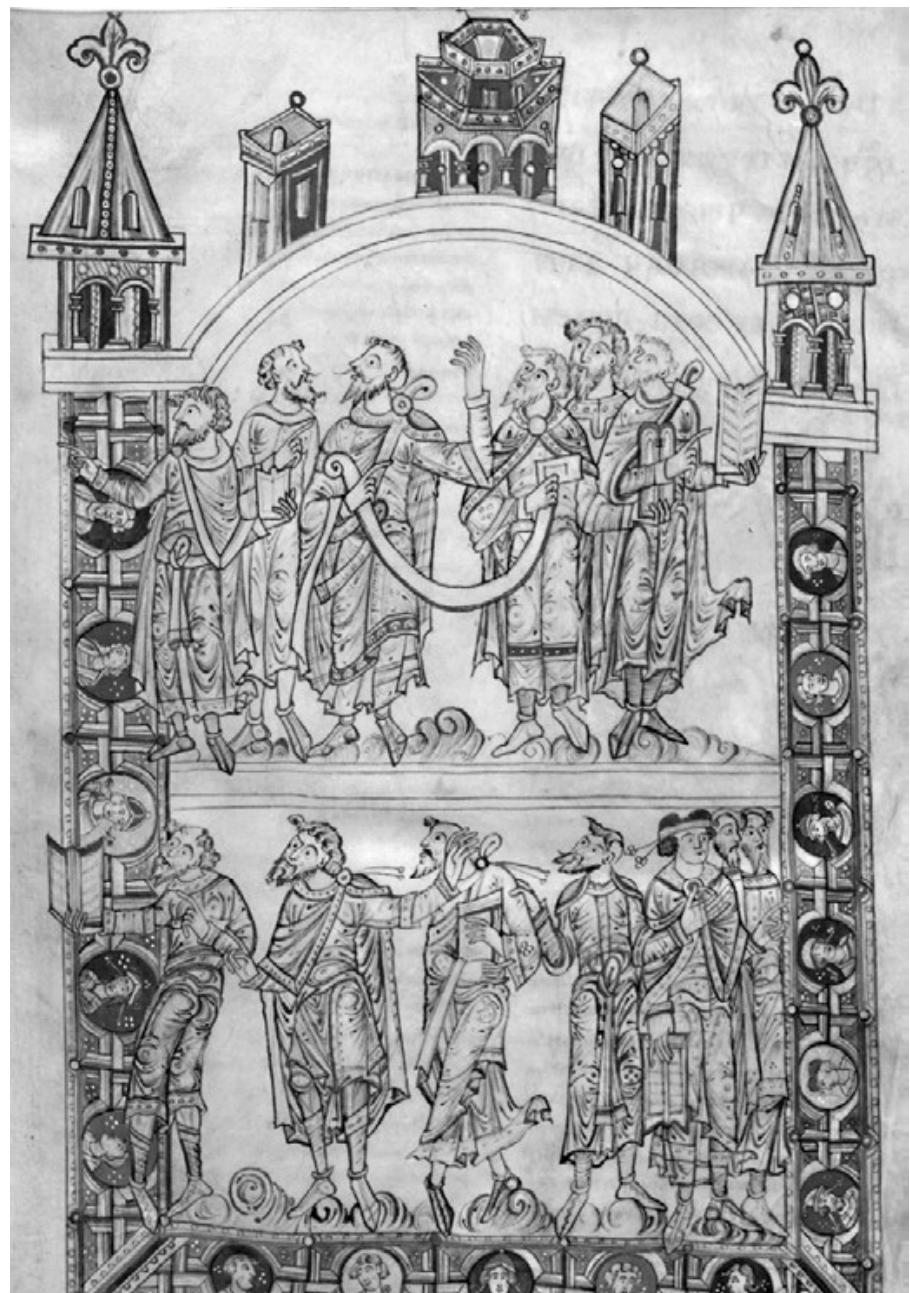

Fig. 6: Firenze, Biblioteca Medicea-Laurenziana, Plut. 12.17, fol. 4r. (C) Biblioteca Medicea-Laurenziana.

È possibile stabilire l'oggetto di questa disputa? Secondo Kenneth B. Steinhauser, l'unico ricercatore che ha sviluppato un' analisi globale del ciclo che orna questo manoscritto, si tratterebbe di una disputa sulla natura dell'anima. Quest' interpretazione deriva da quella della prima miniatura della serie, e in particolare dalla discussiones della psicostasia che, secondo lo studioso, costituirebbe l'oggetto della scena rappresentata due fogli dopo. Non soltanto. A suo avviso: «This one scene in two full page miniatures describes the major

art. cit., p. 579; Wittekind, «Die Illustration von Augustinustexten...», art. cit., e Cosma - Da Gai - Pittiglio, Iconografia agostiniana, cit. 
theme of Augustine's De ciuitate Dei, the encounter of Christianity with Neoplatonic philosophy ${ }^{27}$. Quest'ipotesi, pur seducente, presenta alcuni inconvenienti. Da un punto di vista iconografico, il dispositivo che essa presta alla serie di fogli miniati, e precisamente il fatto che il soggetto della disputa dei ff. $3 \mathrm{v}$ e $4 \mathrm{r}$ sia rappresentato al foglio $1 \mathrm{v}$, non è affatto corrente nel linguaggio figurativo di quest'epoca. Anche in seguito, quando nel xv secolo le miniature ritraggono tanto l'oggetto della discussione quanto la discussione stessa, i diversi soggetti sono generalmente accolti nel medesimo spazio miniato e corredati da una serie di accorgimenti -rubriche e gesti- che servono a guidare alla corretta interpretazione della relazione dei soggetti rappresentati. In sede dottrinaria, si noterà che se l'intenzione di chi ha elaborato queste immagini era di rappresentare la dottrina agostiniana dell'anima di contro a quella neo-platonica della metempsicosi, sarebbe alquanto singolare che per farlo scegliesse il soggetto della psicostasia che, come si è osservato, non è familiare alla teologia agostiniana e piuttosto il portato dell'aggiornamento di quella secondo le coordinate definitesi tra XI e XII secolo. Non da ultimo, e con gli occhi rivolti alla storia della ricezione del trattato agostiniano, mentre la ricezione moderna ama sottolineare il dialogo di Agostino con la filosofia antica, non altrettanto può dirsi per la ricezione medievale e confesso di non conoscere nessuna fonte medievale che definisca il «tema principale» del $D e$ civitate Dei in termini analoghi a quelli illustrati da Steinhauser ${ }^{28}$.

\section{Il ciclo iconografico del Plut. 12, 17 e le due città agostiniane}

Mi sembra dunque opportuno rinunciare alla possibilità d'identificare l'oggetto specifico della disputa rappresentata ai fols. $3 \mathrm{v}$ e $4 \mathrm{r}$, e cercare invece di comprendere l'insieme del ciclo iconografico di questo manoscritto alla luce delle significazioni che nel medioevo erano attribuite al trattato agostiniano, nonché delle attualità di tali significazioni nel contesto specifico

\footnotetext{
${ }^{27}$ Steinhauser, «Augustin moralisé...», art. cit., p. 582.

${ }^{28}$ Non trovo peraltro convincente il parallelo con le Bibles moralisées proposto da questo studioso: elaborazioni dell'inizio del XIII secolo, di fattura parigina, comportanti una selezione del testo, il suo commento e la sua traduzione in lingua vernacolare, e destinate a un pubblico laico si tratta di una tipologia libraria e d'illustrazione del tutto diversa da quella del De civitate Dei della biblioteca medicea. Per un quadro d'insieme, cfr. John Lowden, The Making of the Bibles Moralisées, University Park (PA), Penn State Press, 2000, 2 voll. e Babette Hellemans, La Bible Moralisée: une ouvre à part entière: Temporalité, sémiotique et création au XIII siècle, Turnhout, Brepols, 2010. Steinheuser propone inoltre di interpretare alla luce di questo tema maggiore anche le lettere istoriate nel corpo del codice. Trattandosi di una serie non completa, disomogenea (le lettere istoriate aumentano nella seconda parte del codice la cui ornamentazione è globalmente più curata e ricca), nonché di soggetti talvolta difficili da decifrare e mancando un'analisi codicologica che dettagli le campagne di lavoro, preferisco rinunciare all'idea di «illustrative program» (Steinhauser, «Augustin moralisé...», art. cit., p. 580) e rinviare l'analisi di quest'aspetto ad altra sede.
} 
di elaborazione di queste immagini. Ora, come Agostino spiega nell'estratto delle Retractationes che figura da prologo al De civitate Dei in quasi tutta la tradizione manoscritta (e così nel Plut. 12, 17, fol. 5r), l'opera tratta delle due città e per ragioni di spazio è intitolata alla migliore tra di esse, la civitas $D e i^{29}$. Il brano è celebre presso i lettori del trattato, tanto che il vescovo di Lione Remigio (m. 875) lo ripete per giustificare il titolo del suo De praedistinatione sanctorum ${ }^{30}$, e le due città sono abitualmente citate quando si tratta di descrivere il contenuto del trattato di Agostino ${ }^{31}$.

\subsection{Il saeculum e il trionfo della civitas Dei}

Soffermiamoci allora nuovamente sulle prime due miniature e sul rapporto che intercorre tra loro. Come si è detto, la loro successione dà a vedere e in qualche modo performa, sotto le mani e gli occhi di chi sfogli il codice, lo scarto tra terreno e celeste, tra storia ed eterno, tra immanenza e trascendenza. Più elementi enfatizzano queste connotazioni rispettive. Nella prima miniatura [fig. 1], il decoro vegetale della cornice, la scena rurale del registro inferiore, l'albero che inaugura il mediano e la singolare ambientazione campestre della psicostasia, cui difficilmente si potrebbe attribuire un'intenzione paesaggistica, acquistano senso laddove si ammetta che qui si volesse rappresentare appunto la terrestrità. Viceversa, e come non serve ricordare, il riferimento alla Gerusalemme della visione giovannea della cornice, la seconda parusia del Cristo, la pletora degli eletti, nonché lo sfondo decorato da stelle in tutti i comparti della seconda miniatura insistono sulla natura celeste della città di Dio [fig. 4].

${ }^{29}$ Aug. Hipp., Retract. II 43, 2, ed. A. Mutzenbecher, in Corp. Christ. Ser. Lat. 57, Turnhout, Brepols, 1984, 1. 27: «Duodecim ergo librorum sequentium primi quattuor continent exortum duarum ciuitatum, quarum est una dei altera huius mundi, secundi quattuor excursum earum siue procursum, tertii uero qui et postremi debitos fines. Ita omnes viginti et duo libri, cum sint de utraque ciuitate conscripti, titulum tamen a meliore acceperunt, ut De ciuitate Dei potius uocarentur» (trad. it. cit., p. 4: «Dei dodici libri che seguono dunque i primi quattro contengono l'origine delle due città, una di Dio e l'altra del mondo; gli altri quattro, il loro svolgimento o sviluppo; i quattro successivi, che sono anche gli ultimi, il fine proprio. Sebbene tutti i ventidue libri riguardano l'una e l'altra città, hanno tuttavia derivato il titolo dalla migliore. Perciò è stata preferita l'intestazione La città di Dio»).

${ }^{30} \mathrm{Cfr}$. Remigius Lugdunensis, De tribus epistolis xxxv, in Patr. Lat. 121, coll. 1047B-C: «Et ideo liber ille de praedestinatione sanctorum titulatur, non quia et de praedestinatione reproborum nihil ibi dicitur, sed quia ex meliori parte nomen accepit, sicut et in libris de Ciuitate Dei, utique de utriusque ciuitatibus, et Dei, scilicet, et diaboli pariter et multipliciter disputat; et tamen ex meliori parte libros de Ciuitate Dei uoluit appellare».

${ }^{31}$ Basti il riferimento a Otto Frisingensis, Chronica sive Historia de duabus civitatibus, Prologus, ed. A. Hofmeister, München, 1912 («MGH, SS rer. Germ.», 45), p. 8: «alter [scilicet Augustinus] de gloriosae ciuitatis Dei exortu siue progressu debitisque finibus, quomodo inter mundi ciues semper profecerit, quique eius ciues uel principes quibus principum seu ciuium seculi temporibus extiterint, acutissime disertissimeque disputauit». 
Ricorrendo al lessico agostiniano e medievale, il soggetto della prima miniatura si potrà allora identificare con l'hoc saeculum, con questo mondo e con questo tempo in quanto strutturalmente finiti e sottoposti alla morte, ossia destinati a esaurirsi per lasciare il posto al trionfo della città di Dio alla fine dei tempi, ritratto appunto nella miniatura seguente. La corretta distinzione dell'hoc saeculum dalla dimensione trascendente che è della civitas Dei, così come la corretta articolazione del loro rapporto sono in effetti argomenti fondamentale del trattato di Agostino. Facendo leva su di essi, Agostino disfa l'idolatria dei pagani, che venerano gli dei per assicurarsi una felicità temporale impossibile da conseguire, ma anche l'ideologia statuale romana che si fonda sulla confusione tra ciò che è di questo saeculum e ciò che appartiene all'altro. Diversamente da costoro, il De civitate Dei insegna ai buoni cristiani l'importanza di questa distinzione e, ben articolandola, a sopportare pazientemente le sofferenze della storia in vista della vita che verrà.

Più interessante ai nostri fini e come già accennato nell'introduzione, la distinzione e articolazione di saeculum e aldilà si lascia descrivere, sempre nel lessico agostiniano e medievale, attraverso le immagini delle due città, di modo che la definizione di civitas terrena (o diaboli, e le sue incarnazioni storiche) significa talvolta la mondanità tout court. Già ai tempi del De catechizandis rudibus e nel commento ai salmi, Agostino glossa il nome di Babilonia come «confusione di questo secolo/mondo/tempo», ampliando così il riferimento alla nozione di confusio abitualmente allegato per l'interpretatio nominis di Babilonia in memoria della confusione babelica ${ }^{32}$. Altrove definisce la città negativa sinteticamente come civitas huius saeculi ${ }^{33}$. Questa stessa accezione della civitas terrena (o diaboli) si ritrova, benché solo a tratti, nel De civitate Dei e, come notato da un fine lettore, talvolta «dans l'esprit d'Augustin, la notion de saeculum a tendance à s'identifier avec celle de civitas terrena» ${ }^{34}$.

${ }^{32}$ Cfr. Aug. Hipp., De cat. rud. 21, 37, ed. J.-B. Bauer, Corp. Christ. Ser. Lat. 46, Turnhout, Brepols, 1969, 1. 33: «...ab istius saeculi confusione tamquam de captiuitate Babyloniae...»; Enarr. in ps. 36, 2, ed. E. Dekkers e J. Fraipont, Corp. Christ. Ser. Lat. 38-40, Turnhout 1956, vol. 38, 1. 2: «...in ista vita, in ista confusione saeculi huius, in ista Babylonia...».

${ }^{33}$ Cfr. Aug. Hipp. Enarr. in ps. 125, 3, ed. cit., vol. 40, 1. 18: «Babylonia est enim mundus iste; Babylonia enim interpretatur confusio [...] Confusio ergo est ista tota vita rerum humanarum, non pertinens ad Deum»; ivi 145, 20, ed. cit., vol. 40, 1. 7: «... est certe ciuis de Sion, non de Babylonia; id est, non de ciuitate peritura huius saeculi, sed de Sion ad tempus laborante et peregrinante, in aeternum autem regnatura».

${ }^{34}$ Così Goulven Madec nella sua recensione al libro di Robert A. Markus, Saeculum. History and Society in the Theology of Saint Augustine, Cambridge, Cambridge University Press, 1970, in Revue des Etudes Augustiniennes 17:3-4 (1971), pp. 385-377, a p. 387. Per le occorrenze di questo tipo nel trattato maggiore, si consideri ad esempio De civ. Dei 18, 1, ed. cit., vol. 48, p. 592, 1. 1: «De ciuitatum duarum, quarum Dei una, saeculi huius est altera, quantum ad hominum genus pertinet, etiam ista peregrina...» (trad. it. cit., p. 934: «delle due città, una di Dio, l'altra del tempo, in cui convive, per quanto attiene al genere umano, anche la celeste in esilio»), sebbene poco oltre precisi (ivi 18, 18, ed. cit., vol. 48, p. 608, 
Tale uso delle due città si perpetua nella tradizione medievale. Tra i molti esempi, sarà interessante ricordare come Honorius Augustudonensis sviluppava questo argomento in conclusione di un sermone sulle due città incluso nel suo Speculum Ecclesiae, una raccolta omiletica dell'inizio del XII secolo dedicata ai «fratres Cantuariensis ecclesiae» (da identificarsi forse con i monaci di Christ Church, presso i quali l'autore aveva trascorso un periodo di studio $)^{35}$. Dopo aver dettagliato l'opposizione delle due città in vari modi, nella perorazione finale, Honorius si appella al topos agostiniano della doppia morte (fisica e spirituale):

Et quia homo de gloria in miseriam pulsus in labore nascitur, in dolore nutritur, omne quod laborat ob duas causas laborat, ut videlicet malum effugiat et bonum habeat. Quid autem morte pejus, quid vita melius? Quod ergo homo arat, seminat, metit, fructus colligit, vel aliud quid laboris assumit, hoc ideo utique facit, ut malum, scilicet mortem, evadat, et bonum, videlicet vitam, habeat. Sed hoc frustra conatur, cum omnis homo ex necessitate quandoque moriatur, sicque multis miser miseriis repletur, dum per laborem mortem incurret qui laborabat ne moreretur. Igitur, dilectissimi, cum hoc malum miserabile sit omnibus inevitabile, totis viribus, laboremus ut summum malum, scilicet, aeternam mortem, evadamus, et summum bonum, videlicet aeternam vitam, sine fine habeamus, cum Christus rex noster, cum ventilabro veniens, paleas a granis segregabit, et paleas, id est reprobos, per ultimum judicium igni inextinguibili comburens, triticum, id est electos, in horreum coeleste congregabit, cum fines Hierusalem pacem ponens, regnum Deo Patri tradiderit et Deus omnia in omnibus erit. Qui sit benedictus in saecula ${ }^{36}$.

Il lettore sarà colpito dal campo metaforico dei lavori agricoli, in primis l'aratura, cui Honorius ricorre per figurare la sofferenza dell'essere nel tempo

11. 2-3): «Et quid dicemus, nisi de medio Babylonis esse fugiendum? Quod praeceptum propheticum (cfr. Is 48,20 ) ita spiritualiter intelligetur, ut de huius saeculi ciuitate, quae profecto et angelorum et hominum societas impiorum est...» (trad. it. cit., p. 955: «Dico soltanto che bisogna fuggire di mezzo a Babilonia. Questo avvertimento del profeta va inteso in senso spirituale per fuggire dalla città del tempo, che è la società degli angeli e degli uomini infedeli...»).

${ }^{35}$ Questa dedica figura nel prologus apposto ai manoscritti di Munich, Bayerische Bibl. clm 7700 (XII sec.) e S. Florian, Stiftbibl., cod. XI 252 (XII-XIII sec.) ed è generalmente accolta come autentica dagli studiosi. Sulla vita, assai discussa di questo personaggio, si vedano i vari interventi di Marie-Odile Garrigues e, da ultimi in ordine cronologico, «L'anonymat d'Honorius Augustodunensis», in Studia monastica, 25 (1983), pp. 1-71 e Ead., «L'œuvre d'Honorius Augustodunensis. Inventaire critique», in Abhandlungen der Braunschweigischen Wissenschaftlichen Gesellschaft, 38 (1986), pp. 7-138 e 39 (1987), pp. 113-128; e i lavori coevi di Valérie I. J. Flint poi confluiti in Authors of the Middle Ages, vol. II, 5-6: Peter Abelard (Constant J. Mews) and Honorius Augustodunensis of Regensburg (Valérie I. J. Flint), Aldershot, Variorum 1995.

${ }^{36}$ Honorius Augustodunensis, Speculum Ecclesiae, sermo In conventu Populi, in Patr. Lat. 172, coll. $1100 \mathrm{~A}-\mathrm{C}$. 
e il riferimento al giudizio che tutti ci attende, analoghi al repertorio di immagini della prima miniatura del manoscritto, così come la conclusione topica sulla Gerusalemme celeste, effigiata nella miniatura seguente. Altri elementi dell'iconografia di queste tavole riconducono allo stesso contesto culturale e ideologico, e in particolare l'insistenza sulla connotazione ecclesiale della civitas Dei, con l'enfasi apposta sulla figura allegorica della Ecclesia in cortocircuito analogico con la figura mariale. La prossimità tra discorso verbale e figurativo investe anche in modo di articolare il rapporto tra queste entità: la civitas terrena-saeculum e la civitas Dei trionfante non sono opposte frontalmente bensì è messo in luce lo scarto che separa una dimensione dall'altra e una serie di dettagli sottolineano la possibilità di passare (o meglio di 'trapassare') dall'una all'altra. Non solo la mise en page, ma anche l'anima che, accolta nelle braccia di un angelo, esulta nella prima miniatura e la porta sì sorvegliata ma aperta della civitas Dei della seconda evidenziano questa connessione e invitano il fedele ad effettuarla sfogliando il codice.

\subsection{L'opposizione delle due città in questo mondo}

Diversamente dai precedenti, gli ultimi due fogli miniati rispondono, oltre la globale simmetria -due miniature affrontate, della medesima grandezza e incorniciate entrambe da una struttura architettonica-, a una strategia di differenziazione e opposizione. L'ordine gerarchico della comunità ritratta nella prima miniatura [fig. 5] si oppone al caos, anche apertamente conflittuale, che regna nell'altra [fig. 6]. Allo stesso modo, con il solo codice che Agostino mostra ai suoi interlocutori -e vi si potrà riconoscere lo stesso trattato- contrastano i molti scritti impugnati da costoro, così come contrasta con l'auctoritas salda e ossequiosamente riconosciuta del Padre della Chiesa la molteplicità delle auctoritates, tra loro in disaccordo, della seconda miniatura.

$\mathrm{Si}$ tratta nuovamente di opposizioni agostiniane e correnti in quel $\mathrm{De} \mathrm{ci-}$ vitate Dei che si compiace di far esplodere le contraddizioni che travagliano il complesso delle filosofie antiche in modo da meglio confortare la dottrina cristiana. Non soltanto. Anche in questo caso, si tratta di un'opposizione che Agostino connette con quella delle due città. La celebre conclusione del XIV libro del De civitate Dei illustra l'antagonismo tra amor sui e amor Dei in modo da coglierne la dimensione anche culturale e dottrinaria:

Fecerunt itaque ciuitates duas amores duo, terrenam scilicet amor sui usque ad contemptum dei, caelestem uero amor dei usque ad contemptum sui.Denique illa in se ipsa, haec in domino gloriatur. [...] Ideo que in illa sapientes 
eius secundum hominem uiuentes aut corporis aut animi sui bona aut utriusque sectati sunt, aut qui potuerunt cognoscere deum, "non ut deum honorauerunt aut gratias egerunt, sed euanuerunt in cogitationibus suis, et obscuratum est insipiens cor eorum"; "dicentes se esse sapientes (id est dominante sibi superbia in sua sapientia sese extollentes) stulti facti sunt et inmutauerunt gloriam incorruptibilis dei in similitudinem imaginis corruptibilis hominis et uolucrum et quadrupedum et serpentium (ad huiusce modi enim simulacra adoranda uel duces populorum uel sectatores fuerunt), et coluerunt atque seruierunt creaturae potius quam creatori, qui est benedictus in saecula"; in hac autem nulla est hominis sapientia nisi pietas, qua recte colitur uerus deus, id expectans praemium in societate sanctorum non solum hominum, uerum etiam angelorum, ut sit deus omnia in omnibus ${ }^{37}$.

Alla luce di questo riferimento si potrà inoltre comprendere il dettaglio dei medaglioni che ornano la cornice dell'ultima miniatura [fig. 6]. Questo trattamento iconografico non è sconosciuto all'arte insulare di quest'epoca ma neanche corrente; non rientra cioè in un repertorio puramente ornamentale (sempre che tale definizione abbia un senso) ed è in genere depositario di significato come avviene, ad esempio, nella I decorata con ritratti di Cristo e di santi che inaugura una raccolta di vite dei santi in un manoscritto redatto a Canterbury alla prima metà del XII secolo [fig. 7 $]^{38}$. La serie di medaglioni-ritratti acquista un significato laddove si tenga presente la polemica agostiniana contro l'idolatria -che, come emerge anche nella citazione precedente, riveste nel discorso di Agostino un campo semantico assai vasto, designando generalmente la devozione rivolta alla creatura invece che al Creatore-e il modo in cui questa polemica è connessa alla problematica cristiana dell'imago. Non è un caso che Agostino citi nel De civitate Dei un versetto del Salmo 72, che

\footnotetext{
${ }^{37}$ Aug. Hipp., De civ. Dei 14, 28, ed. cit., vol. 48, p. 451, 11. 1-12; trad. it. cit., p. 736: «Due amori dunque diedero origine a due città, alla terrena l'amor di sé fino all'indifferenza per Iddio, alla celeste l'amore a Dio fino all'indifferenza per sé. Inoltre quella si gloria in sé, questa nel Signore. [...] Quindi nella città terrena i suoi filosofi, che vivevano secondo l'uomo, hanno dato rilievo al bene o del corpo $o$ dell'anima o di tutti e due. Coloro poi che poterono conoscere Dio, "non lo adorarono e ringraziarono come Dio, si smarrirono nei propri pensieri e fu lasciato nell'ombra il loro cuore stolto perché credevano di esser sapienti", cioè perché dominava in loro la superbia in quanto si esaltavano nella propria sapienza. Perciò "divennero sciocchi e sostituirono alla gloria di Dio non soggetto a morire l'immagine dell'uomo soggetto a morire e di uccelli e di quadrupedi e di serpenti" $e$ in tali forme di idolatria furono guide o partigiani della massa. "Così si asservirono nel culto alla creatura anziché al Creatore che è benedetto per sempre" $(R m$ 1, 21-23.25). Nella città celeste invece l'unica filosofia dell'uomo è la religione con cui Dio si adora convenientemente, perché essa attende il premio nella società degli eletti, non solo uomini ma anche angeli, "affinché Dio sia tutto in tutti" $(1$ Cor 15, 28)».

${ }^{38}$ London, British Library, Harley, ms. 624, fol. 94r. Mutuo tale esempio da Dodwell, The Canterbury School of Illumination, cit., tav. 17, p. 28, che segnala l'origine ottoniana di questo motivo e il ricorrere del decoro a «interlocking bars» anche in altri manoscritti provenienti da Canterbury.
} 


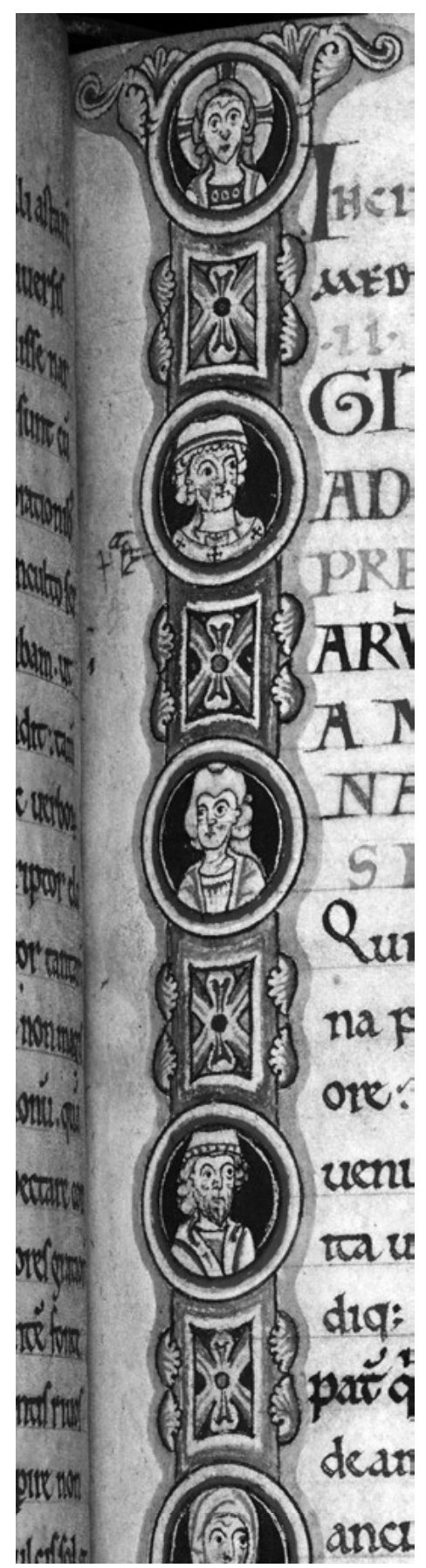

Fig. 7: Londra, British Library, Harley, ms. 624, fol. 94r. (C) British Library. aveva già lungamente commentato nelle Enarrationes, in questi termini: «Et quoniam in hac terra uel in ciuitate terrena magni sibi uidebantur: "Domine, inquit, in ciuitate tua imaginem eorum ad nihilum rediges" $\left.\left(P_{S} 72,20\right)\right\rangle^{39}$. Gli empi membri della civitas terrena, che prediligono la creatura al Creatore e sono perciò idolatri, sono convinti della loro grandezza mondana; pertanto, come degno contrappasso, Dio annichilirà le loro imagines nella civitas Dei.

Questo versetto e l'esegesi di Agostino sono familiari alla tradizione cristiana medievale, ripresi per esempio nei Libri carolini ${ }^{40}$ oltre che nei commenti ai salmi ${ }^{41}$. Si suggerirà allora che la miniatura del fol. $4 \mathrm{r}$ intendesse rappresentare la civitas terrena (o diaboli) in quanto popolata dai falsi sapienti e ornata dalle loro imagines (i medaglioni della cornice), quelle stesse che saranno distrutte nella città di Dio. Sarà a questo punto interessante notare che questo dispositivo, al tempo stesso figurativo e simbolico, è il medesimo che si riscontra in un manoscritto miniato del De civitate Dei della seconda metà del XII secolo proveniente dal monastero benedettino di Santa Maria di Bosau in Sassonia. Lì, la miniatura a piena pagina deputata a rappresentare la civitas terrena (o diaboli) è affrontata alla miniatura della $\mathrm{ci}$ vitas Dei (nello stesso ordine e mise en page che il Plut. 12.17) e racchiusa da una cornice

${ }^{39}$ Aug. Hipp., De civ. Dei 10, 25, ed. cit., vol. 47, 1. 25; trad. it. cit., p. 498: «E poiché si ritenevano grandi in questa terra, ossia nella città terrena, soggiunse: "Signore, tu nella tua città ridurrai al nulla la loro figura"».

${ }^{40} \mathrm{Cfr}$. Opus Caroli regis contra synodum II 3, ed. Ann Freeman, MGH, Conc. 2., Suppl. 1, 1998, p. 241 e ss., da integrare con i saggi raccolti in Ann Freeman, Theodulf of Orléans: Charlemagne's Spokesman against the Second council of Nicaea, Ashgate, Variorum, 2003.

${ }^{41} \mathrm{~A}$ titolo di esempio si veda Haimo Halberstatensis, $\mathrm{Com}$ mentaria in Psalmos 72, 20, in Patr. Lat. 116, coll. 441B- 441C. 
architettonica ornata da medaglioni raffiguranti le divinità pagane, in richiamo più aperto all'idolatria ${ }^{42}$.

Soprattutto, il fatto di ritrarre le due città agostiniane sotto quest'angolatura, culturale e dottrinaria, oltre che ben fondato nell'opera che ci si proponeva di illustrare possiede anche una certa attualità nel contesto di elaborazione di questo manoscritto. Nella commendatio del già citato Speculum Ecclesiae, Honorius giustifica la sua compilazione come da prassi ma arricchisce la topica consueta facendo appello alle due città agostiniane, ricordate attraverso le loro due rappresentanti storiche per antonomasia, Gerusalemme e Babilonia:

Rogo autem laborantes in caribdi saecularis phylosophiae ne despiciant hoc pitaciolum legis divinae, scientes hoc non sibi sed civibus Hierusalem, scilicet humilibus, conscriptum, quibus solummodo et supernum regnum ut filiis est promissum. Cives autem Babyloniae, videlicet superbi, habunde sunt a suis praeceptoribus instructi. Horum doctrinis innitantur quorum et vitam summopere imitantur. Et ideo qui naenias opinionis mundanae dispositionis amplectuntur, legant Platonem; quos cavillare delectat, discant Aristotelem; bella amantes habent Maronem; libidini vacantes, Nasonem; discordes incitat Lucanus et Stacius; petulantes instruit Horacius et Terentius; sed quia horum nomina de libro viventium sunt deleta, non memor ero nominum eorum per labia mea $^{43}$.

L'autore indirizza il suo lavoro ai cives Hierusalem, e invita sprezzantemente coloro che si affaticano nello studio della filosofia secolare, i cives

${ }^{42}$ Pforta, Bibliothek der Kgl. Landes-schule, ms. lat. A 10, ff. 2v-3r, sul quale cfr. Brilli, «As Formas da História...», art. cit., pp. 137-144. Il raffronto non intende suggerire una parentela tra questi testimoni che è lungi dall'essere dimostrabile.

${ }^{43}$ Honorius Augustodunensis, Speculum Ecclesiae, Commendatio huius operis, in Patr. Lat. 172, coll. 1085D-1086D. Un passo di simile tenore si legge nella prefazione al Gemma animae dello stesso autore (cfr. Patr. Lat., coll. 513-514: «Plerosque vesania captos piget me mente considerare, quos non pudet abominanda poetarum figmenta ac captiosa philosophorum argumenta summo conamine indagare, quae mentem ideo abstractam vitiorum nexibus insolubiliter solent innodare, religionem autem Christianae professionis penitus ignorare, per quam animam liceat perenniter cum Deo regnare. Cum sit summae dementiae jura tyranni velle scire, et edicta summi imperatoris nescire, atque ea quae quotidie necessario facias non intelligere. Porro quid confert animae pugna Hectoris, vel disputatio Platonis, aut carmina Maronis, vel neniae Nasonis, qui nunc cum consimilibus suis strident in carcere infernalis Babylonis, sub truci imperio Plutonis. Dei autem sapientia maxima gloria hunc cumulat, qui prophetarum et apostolorum facta et scripta investigando jugiter ruminat, quos nunc in coelestis Hierusalem palatio cum rege gloriae exsultare nemo dubitat. Sapientum namque judicio tantum differt a non intelligente intelligens, quantum a caeco videns. Qui enim non intelligit quae agit, est ut caecus, qui nescit quo vadat; et ut Tantalus in mediis undis siti depcrit. Et licet simplicitas fidelium Deo nostro placeat, tamen intelligentiam sapientum quantum lucem prae tenebris approbat. Ob hanc causam ut jussistis, libellum De divinis officiis edidi, cui nomen Gemma animae indidi. Quia videlicet veluti aurum gemma ornatur, sic anima divino officio decoratur»). 
Babylonis, a considerare che il suo «breve scritto» (pitaciolum) non li concerne affatto. Li rinvia dunque ai loro precettori -esemplificati dalla lista degli autori pagani-, dei quali lui invece non farà menzione poiché i loro nomi non figurano nel libro dei viventi, dei redenti e salvi in Cristo. È stato suggerito che questo passo fosse animato dalla polemica sviluppata dai centri monastici contro le nuove forme di sapere e d'insegnamento caratteristiche, all'inizio del XII secolo, delle scuole cattedrali più aperte allo studio dei classici e delle arti liberali ${ }^{44}$. In mancanza di altri elementi, quest'ipotesi di lettura appare azzardata; nondimeno, il passaggio della commendatio ben certifica la vitalità di questo tema agostiniano in un contesto che, malgrado le dispute che videro opporsi Christ Church e l'abbazia di Saint Augustine, è culturalmente assai prossimo a quello di elaborazione del De civitate Dei della biblioteca medicea.

Si potrà dunque riconoscere in questa doppia pagina una nuova rappresentazione delle due città agostiniane effigiate ora entrambe in questo mondo e contrapposte in ragione del loro diverso orientamento, quell'opzione di amor sui e amor Dei della quale Agostino aveva mostrato le ricadute anche in sede culturale e dottrinaria. A differenza della coppia precedente, i dettagli così come la mise en page sottolineano non tanto lo scarto quanto piuttosto la contrapposizione tra i soggetti rappresentati; e si noterà che l'ordine (prima la civitas Dei, poi la terrena o diaboli) risponde ora a una polarizzazione simbolica dello spazio, organizzato in modo speculare rispetto alle coordinate dell'osservatore come avviene nel cosiddetto campo araldico e nel campo escatologico, e che è la stessa alla quale rispondo tutte le immagini delle due città reperibili nel corpus dei manoscritti miniati del trattato agostiniano, se contrapposte orizzontalmente ${ }^{45}$.

\footnotetext{
${ }^{44}$ Cfr. Eva Matthews-Sanford, «Honorius, Presbyter and Scholasticus», in Speculum, $23: 3$ (1948), pp. 397-425, a p. 423: «His attack on the 'citizens of Babylon' and the pagan authors in whom they delighted, in the preface of the Speculum Ecclesiae, was probably aimed at the clever young men who returned from their studies in France to disturb the peace of the German schools by the contentious scholasticism they had learned under William of Champeaux or Anselm of Laon». Il riferimento apposto a questo passo (ivi, nota 136) a Joseph de Ghellinck, Le mouvement théologique du XII siècle, Paris, J. Gabalda, 1914, p. 87, sembra essere frutto di una svista e, né nell'edizione del 1914 né nella seconda rivista e incrementata del 1948 (Paris-Bruxelles, Editions «De Tempel»), Ghellinck non si riferisce al prologo dello Speculum ecclesiae.

${ }^{45}$ I soggetti positivi sono posti alla sinistra dell'osservatore (ossia alla destra di chi tenga uno scudo, o del Cristo nelle rappresentazioni del Giudizio universale), mentre i negativi occupanto la parte opposta. Per la definizione di «campo araldico», cfr. Michel Pastoreau, Traité d'Héraldique, Paris, Picard, 1979, p. 99. Per i dati relativi alla totalità del corpus cfr. Brilli, Una vicina città..., op. cit., vol. 2, pp. 661-665.
} 


\section{Conclusioni: poetica verbale e poetica figurativa delle due città agostiniane}

Considerando il ciclo iconografico del Plut. 12, 17 nel suo insieme, sembra dunque che i suoi ideatori abbiano inteso rappresentare due volte la coppia delle due città agostiniane quali le conoscevano. Innanzitutto, si sono concentrati sulla dialettica tra mondano ed eterno, tra la civitas terrena-saeculum inesorabilmente orientata alla morte, al giudizio e la perfezione della civitas Dei; quindi, in questo mondo, hanno focalizzato l'opposizione tra due modi di vita e tra due culture, quella ispirata alla vera sapienza e ossequiosa della giusta auctoritas e quella, superba e conflittuale, dei rappresentanti antichi e coevi della sapienza pagana.

L'ipotesi del raddoppiamento del soggetto delle due città può suonare poco economica o poco armoniosa. Credo nondimeno che sia la migliore per rendere conto dell'insieme degli elementi iconografici che si sono evidenziati e la più calzante al contesto di elaborazione di queste immagini. Inoltre, assumendola, si può forse cogliere un aspetto fondamentale delle due città agostiniane e della loro diversa resa in sede verbale e in sede figurativa.

Questo raddoppiamento può infatti essere inteso come una risposta all' esigenza di tradurre visivamente la pluralità di significazioni che le due città rivestono nel discorso agostiniano e medievale. Come accennato nell'introduzione, le due città, e la città negativa soprattutto, non sono semplicemente polisemiche ma anche equivoche. Ciò nella misura in cui dietro e attraverso queste immagini discorsive Agostino, e così il la tradizione medievale, combinano delle dualità -quella ontologica di transeunte $v s$ eterno e quella etico-escatologica di amor sui vs amor Dei-che, per quanto concettualmente imbricate, non sono tuttavia in alcun modo riducibili l'una all'altra nell'orizzonte monoteista cristiano. Ne risulta che la civitas terrena (o diaboli) designa ora la storicità post lapsus tutta, ora la sola compagine di coloro che si votano volontariamente al male. Quest'equivocità è regolarmente messa a frutto da Agostino e nella tradizione medievale, ma anche dissimulata. La diacronia propria al discorso verbale consente infatti di utilizzare le medesime etichette assumendole ora in un senso ora nell'altro in modo tale che, di slittamento in slittamento, il discorso mantiene una parvenza di coerenza.

Diversamente, il discorso figurativo è chiamato a scegliere, nella sincronia di un'immagine, una struttura iconografica e ad attenervisi. L'equivocità dell'immagine discorsiva delle due città diviene pertanto problematica nel momento in cui la si voglia trasporre nel codice figurativo che, straordinariamente inventivo laddove si tratti di trasporre la polisemia -come ben testimonia, nello stesso Plut. 12.17, l'immagine della civitas Dei trionfante che 
sintetizza mirabilmente i vari referenti di questa nozione (Gerusalemme celeste, assise dei beati, ecclesia ed Eden a un tempo)-, si trova invece in questo caso nell'impasse di dover scegliere se rappresentare il confronto tra le due città nei termini del rapporto tra immanenza e trascendenza oppure di quello tra due opposte attitudini etiche e destinazioni escatologiche.

Posti di fronte a questo problema, gli ideatori della raffinata iconografia del De civitate Dei della biblioteca medicea rifiutano di sacrificare un'accezione delle due città in favore dell'altra. Optano di conseguenza per il raddoppiamento del soggetto, che effigiano ora sotto il profilo che ho chiamato ontologico, ora sotto quello etico-escatologico, qui ulteriormente precisato nel senso dell'opposizione culturale e dottrinaria che doveva essere loro specialmente cara. Così facendo, tuttavia, questa iconografia rende tangibile il problema posto dalle due città agostiniane per chi sappia riconoscere, oltre le immagini materiali, la rete delle immagini mentali che le hanno occasionate, così come le specificità proprie al discorso verbale e figurativo, ossia le loro differenti poetiche. 
$\operatorname{sen}$

RIASSUNTO: Quest'articolo sottolinea la dimensione poetica delle due città agostiniane, civitas Dei e terrena (o diaboli), per poi esaminare il suo diveso trattamento in sede verbale sia in sede figurativa. Come termine di comparazione è preso come esempio un celebre manoscritto miniato de De civitate Dei (Plut. 12.17). In accordo tanto con il trattato agostiniano quanto con la sua ricezione medievale, l'articolo offre nuova e originale interpretazione delle quattro tavole miniate che aprono questo manoscritto.

ABSTRACT: This article points out the poetic dimension of the two cities defined by S. Augustine, the civitas Dei and the civitas terrena (or diaboli); then it considers the different treatment of such a dimension in the verbal and in the visual code. In order to develop this comparison the author examines a well-known illuminated manuscript of the De civitate Dei (Plut. 12.17; Canterbury? 1120-1130). In keeping with the Augustinian treatise as well as with its medieval reception, the article offers a new and original insight of the four depicted folios, which open this manuscript.

Parole chiave: Agostino. De civitate Dei. Civitas terrena. Civitas diaboli. Saeculum. Canterbury. Polisemia. Equivocità. Poetica verbale. Poetica figurativa. Antitesi.

Keywords: Augustin. De civitate Dei. Civitas terrena. Civitas diaboli. Saeculum. Canterbury. Polisemy. Ambiguity. Verbal poetry. Visual poetry. Antithesis. 